\title{
Asymptotic normality for inference on multisample, high-dimensional mean vectors under mild conditions
}

\author{
Makoto Aoshima • Kazuyoshi Yata
}

Received: date / Accepted: date

\begin{abstract}
In this paper, we consider the asymptotic normality for various inference problems on multisample and high-dimensional mean vectors. We verify that the asymptotic normality of concerned statistics is proved under mild conditions for high-dimensional data. We show that the asymptotic normality can be justified theoretically and numerically even for non-Gaussian data. We introduce the extended cross-data-matrix (ECDM) methodology to construct an unbiased estimator at a reasonable computational cost. With the help of the asymptotic normality, we show that the concerned statistics given by ECDM can ensure consistency properties for inference on multisample and high-dimensional mean vectors. We give several applications such as confidence regions for high-dimensional mean vectors, confidence intervals for the squared norm and the test of multisample mean vectors. We also provide sample size determination so as to satisfy prespecified accuracy on inference. Finally, we give several examples by using a microarray data set.
\end{abstract}

Keywords Asymptotic normality · Confidence region · Cross-data-matrix methodology · Large $p$ small $n$. Microarray · Two-stage procedure

Mathematics Subject Classification (2000) 62H10 - 62L10 - 60F05

\section{Introduction}

A common feature of high-dimensional data is that the data dimension is high, however, the sample size is relatively small. This is the so-called "HDLSS" or

Makoto Aoshima

Institute of Mathematics, University of Tsukuba, Ibaraki 305-8571, Japan

E-mail: aoshima@math.tsukuba.ac.jp

Kazuyoshi Yata

Institute of Mathematics, University of Tsukuba, Ibaraki 305-8571, Japan

E-mail: yata@math.tsukuba.ac.jp 
"large $p$, small $n$ " situation where $p / n \rightarrow \infty$; here $p$ is the data dimension and $n$ is the sample size. The statistical inference on this type of data is becoming increasingly relevant. Suppose we have independent and $p$-variate populations, $\pi_{i}, i=1, \ldots, k$, having unknown mean vector $\boldsymbol{\mu}_{i}$ and unknown covariance matrix $\boldsymbol{\Sigma}_{i}(\geq \boldsymbol{O})$ for each $\pi_{i}$. Let $\boldsymbol{\theta}=\left(\boldsymbol{\mu}_{1}, \ldots, \boldsymbol{\mu}_{k}, \boldsymbol{\Sigma}_{1}, \ldots, \boldsymbol{\Sigma}_{k}\right)$. We do not assume either the normality of the population distributions or the equality of $\boldsymbol{\Sigma}_{\mathrm{s}}$ as $\boldsymbol{\Sigma}_{1}=\cdots=\boldsymbol{\Sigma}_{k}$. The eigen-decomposition of $\boldsymbol{\Sigma}_{i}(i=1, \ldots, k)$ is given by $\boldsymbol{\Sigma}_{i}=\boldsymbol{H}_{i} \boldsymbol{\Lambda}_{i} \boldsymbol{H}_{i}^{T}$, where $\boldsymbol{\Lambda}_{i}$ is a diagonal matrix of eigenvalues, $\lambda_{i 1} \geq \cdots \geq$ $\lambda_{i p} \geq 0$, and $\boldsymbol{H}_{i}$ is an orthogonal matrix of the corresponding eigenvectors. In this paper, we focus on inference on multisample and high-dimensional mean vectors. Let $\boldsymbol{\mu}=\sum_{i=1}^{k} b_{i} \boldsymbol{\mu}_{i}$, where $b_{i}$ s are known and nonzero scalars. Having recorded i.i.d. samples, $\boldsymbol{x}_{i j}, j=1, \ldots, n_{i}$, of size $n_{i}(\geq 4)$ from each $\pi_{i}$, we define $\boldsymbol{T}_{\mathbf{n}}=\sum_{i=1}^{k} b_{i} \overline{\boldsymbol{x}}_{i n_{i}}$, where $\boldsymbol{n}=\left(n_{1}, \ldots, n_{k}\right)$ and $\overline{\boldsymbol{x}}_{i n_{i}}=\sum_{j=1}^{n_{i}} \boldsymbol{x}_{i j} / n_{i}$.

Chen and Qin (2010) gave a two-sample test in inference on HDLSS data. Aoshima and Yata (2011a,b) developed a variety of inference on HDLSS data such as a given-bandwidth confidence region, a two-sample test, a test of equality of two covariance matrices, classification, variable selection, regression, pathway analysis and so on along with sample size determination for each inference. Yata and Aoshima (2012) provided given-width confidence intervals for the norm of mean vectors. Aoshima and Yata (2011a,b) and Yata and Aoshima (2012) assumed that $\boldsymbol{x}_{i j}=\boldsymbol{H}_{i} \boldsymbol{\Lambda}_{i}^{1 / 2} \boldsymbol{z}_{i j}+\boldsymbol{\mu}_{i}$ for $i=1, \ldots, k ; j=1, \ldots, n_{i}$, where $\boldsymbol{z}_{i j}=\left(z_{i 1 j}, \ldots, z_{i p j}\right)^{T}, E\left(\boldsymbol{z}_{i j}\right)=\mathbf{0}, \operatorname{Var}\left(\boldsymbol{z}_{i j}\right)=\boldsymbol{I}_{p}$ and the fourth moments of each variable in $\boldsymbol{z}_{i j}$ are uniformly bounded. Here, $\boldsymbol{I}_{p}$ denotes the identity matrix of dimension $p$. They considered one of the following three assumptions for $\pi_{i} \mathrm{~s}$ :

(A-i) $\quad \pi_{i}: N_{p}\left(\boldsymbol{\mu}_{i}, \boldsymbol{\Sigma}_{i}\right)$ for $i=1, \ldots, k$

(A-ii) $z_{i s j}, s=1, \ldots, p$, are independent for $i=1, \ldots, k$;

(A-iii) $E\left(z_{i s j}^{2} z_{i t j}^{2}\right)=E\left(z_{i s j}^{2}\right) E\left(z_{i t j}^{2}\right)$ and $E\left(z_{i s j} z_{i t j} z_{i u j} z_{i v j}\right)=0$ for $s \neq$ $t, u, v$, and some regularity conditions given in Aoshima and Yata (2011a).

They assumed the following conditions for $\boldsymbol{\Sigma}_{i} \mathrm{~s}$ :

(A-iv) $\liminf _{p \rightarrow \infty} \lambda_{i p}>0, \limsup _{p \rightarrow \infty} \frac{\operatorname{tr}\left(\boldsymbol{\Sigma}_{i}^{t}\right)}{p}<\infty(t=1,2)$ and $\frac{\operatorname{tr}\left(\boldsymbol{\Sigma}_{i}^{4}\right)}{p^{2}} \rightarrow 0$ as
$p \rightarrow \infty$ for $i=1, \ldots, k$.

Note that (A-i) implies (A-ii). It holds that $E_{\boldsymbol{\theta}}\left(\left\|\boldsymbol{T}_{\mathbf{n}}-\boldsymbol{\mu}\right\|^{2}\right)=\sum_{i=1}^{k} b_{i}^{2} \operatorname{tr}\left(\boldsymbol{\Sigma}_{i}\right) / n_{i}$ $\left(=\Sigma_{\mathbf{n}}\right.$, say). Also, it holds that $\operatorname{Var}_{\boldsymbol{\theta}}\left(\left\|\boldsymbol{T}_{\mathbf{n}}-\boldsymbol{\mu}\right\|^{2}\right)=2 \sum_{i, j} b_{i}^{2} b_{j}^{2} \operatorname{tr}\left(\boldsymbol{\Sigma}_{i} \boldsymbol{\Sigma}_{j}\right) /\left(n_{i} n_{j}\right)$ under (A-i). Let $\boldsymbol{S}_{i n_{i}}=\sum_{j=1}^{n_{i}}\left(\boldsymbol{x}_{i j}-\overline{\boldsymbol{x}}_{i n_{i}}\right)\left(\boldsymbol{x}_{i j}-\overline{\boldsymbol{x}}_{i n_{i}}\right)^{T} /\left(n_{i}-1\right)$ and $\widehat{\Sigma}_{\mathbf{n}}=$ $\sum_{i=1}^{k} b_{i}^{2} \operatorname{tr}\left(\boldsymbol{S}_{i n_{i}}\right) / n_{i}$. It holds that $E_{\boldsymbol{\theta}}\left(\left\|\boldsymbol{T}_{\mathbf{n}}-\boldsymbol{\mu}\right\|^{2}-\widehat{\Sigma}_{\mathbf{n}}\right)=0$ and

$\operatorname{Var}_{\boldsymbol{\theta}}\left(\left\|\boldsymbol{T}_{\mathbf{n}}-\boldsymbol{\mu}\right\|^{2}-\widehat{\Sigma}_{\mathbf{n}}\right)=2 \sum_{i=1}^{k} \frac{b_{i}^{4} \operatorname{tr}\left(\boldsymbol{\Sigma}_{i}^{2}\right)}{n_{i}\left(n_{i}-1\right)}+4 \sum_{i<j} \frac{b_{i}^{2} b_{j}^{2} \operatorname{tr}\left(\boldsymbol{\Sigma}_{i} \boldsymbol{\Sigma}_{j}\right)}{n_{i} n_{j}} \quad(=K$, say $)$.

Let $\widehat{T}_{\mathbf{n}}=\left\|\boldsymbol{T}_{\mathbf{n}}\right\|^{2}-\widehat{\Sigma}_{\mathbf{n}}$. It holds that $E_{\boldsymbol{\theta}}\left(\widehat{T}_{\mathbf{n}}\right)=\|\boldsymbol{\mu}\|^{2}$ and $\operatorname{Var}_{\boldsymbol{\theta}}\left(\widehat{T}_{\mathbf{n}}\right)=K+$ $4 \sum_{i=1}^{k} b_{i}^{2} \boldsymbol{\mu}^{T} \boldsymbol{\Sigma}_{i} \boldsymbol{\mu} / n_{i}$ (= $K_{*}$, say). Then, the following results were obtained by Aoshima and Yata (2011a) and Yata and Aoshima (2012). 
Theorem 1 (Aoshima and Yata, 2011a) Assume (A-i) and (A-iv). Then, it holds that

$$
\frac{\left\|\boldsymbol{T}_{\mathbf{n}}-\boldsymbol{\mu}\right\|^{2}-\Sigma_{\mathbf{n}}}{\sqrt{2 \sum_{i, j} b_{i}^{2} b_{j}^{2} \operatorname{tr}\left(\boldsymbol{\Sigma}_{i} \boldsymbol{\Sigma}_{j}\right) /\left(n_{i} n_{j}\right)}} \Rightarrow N(0,1)
$$

when $p \rightarrow \infty$ and either $n_{i} \rightarrow \infty$ or $n_{i}$ is fixed for $i=1, \ldots, k$, where " $\Rightarrow$ " denotes the convergence in distribution and $N(0,1)$ denotes a random variable distributed as the standard normal distribution.

Theorem 2 (Aoshima and Yata, 2011a) Assume (A-iv) and either (A-ii) or (A-iii). Then, (1) holds as $p \rightarrow \infty$ and $n_{i} \rightarrow \infty, i=1, \ldots, k$.

Corollary 1 (Aoshima and Yata, 2011a) Assume (A-iv) and either (A-ii) or $\left(A\right.$-iii). Then, it holds as $p \rightarrow \infty$ and $n_{i} \rightarrow \infty, i=1, \ldots, k$, that

$$
\frac{\left\|\boldsymbol{T}_{\mathbf{n}}-\boldsymbol{\mu}\right\|^{2}-\widehat{\Sigma}_{\mathbf{n}}}{K^{1 / 2}} \Rightarrow N(0,1) .
$$

Theorem 3 (Yata and Aoshima, 2012) Assume that $n_{i} \boldsymbol{\mu}^{T} \boldsymbol{\Sigma}_{i} \boldsymbol{\mu} / \operatorname{tr}\left(\boldsymbol{\Sigma}_{i}^{2}\right)=$ $o(1), i=1, \ldots, k$. Assume $(A$-iv) and either (A-ii) or (A-iii). Then, it holds as $p \rightarrow \infty$ and $n_{i} \rightarrow \infty, i=1, \ldots, k$, that

$$
\frac{\widehat{T}_{\mathbf{n}}-\|\boldsymbol{\mu}\|^{2}}{K_{*}^{1 / 2}} \Rightarrow N(0,1) .
$$

In this paper, we relax the conditions to verify the asymptotic normality of concerned statistics for high-dimensional data. Then, we apply the asymptotic normality to various inference problems on multisample and high-dimensional mean vectors. In Section 2, we verify that the asymptotic normality of concerned statistics is proved under mild conditions. We show that the asymptotic normality can be justified theoretically and numerically even for non-Gaussian data. In Section 3, we introduce the extended cross-data-matrix (ECDM) methodology, developed by Yata and Aoshima (2013), to construct an unbiased estimator at a reasonable computational cost. In Section 4, with the help of the asymptotic normality, we show that the statistics given by ECDM can ensure consistency properties for inference on multisample and high-dimensional mean vectors. We give several applications such as confidence regions for highdimensional mean vectors, confidence intervals for the squared norm and the test of multisample mean vectors. In Section 5, we provide sample size determination so as to satisfy prespecified accuracy on inference. Finally, in Section 6 , we give several examples by using a microarray data set.

\section{Asymptotic normality under mild conditions}

We assume that

$$
\boldsymbol{x}_{i j}=\boldsymbol{\Gamma}_{i} \boldsymbol{w}_{i j}+\boldsymbol{\mu}_{i} \text { for } i=1, \ldots, k ; j=1, \ldots, n_{i},
$$


where $\boldsymbol{\Gamma}_{i}$ is a $p \times r_{i}$ matrix for some $r_{i}>0$ such that $\boldsymbol{\Gamma}_{i} \boldsymbol{\Gamma}_{i}^{T}=\boldsymbol{\Sigma}_{i}$, and $\boldsymbol{w}_{i j}, j=$ $1, \ldots, n_{i}$, are i.i.d. random vectors having $E\left(\boldsymbol{w}_{i j}\right)=\mathbf{0}$ and $\operatorname{Var}\left(\boldsymbol{w}_{i j}\right)=\boldsymbol{I}_{r_{i}}$. Note that (2) includes the case that $\boldsymbol{\Gamma}_{i}=\boldsymbol{H}_{i} \boldsymbol{\Lambda}_{i}^{1 / 2}$ and $\boldsymbol{w}_{i j}=\boldsymbol{z}_{i j}$. Refer to Bai and Saranadasa (1996), Chen and Qin (2010) and Aoshima and Yata (2013) for the details of the model. Let $\boldsymbol{w}_{i j}=\left(w_{i 1 j}, \ldots, w_{i r_{i} j}\right)^{T}$ for any $i, j$. As for $\boldsymbol{w}_{i j}$, we assume that

(A-v) The fourth moments of each variable in $\boldsymbol{w}_{i j}$ are uniformly bounded, $E\left(w_{i s j}^{2} w_{i t j}^{2}\right)=E\left(w_{i s j}^{2}\right) E\left(w_{i t j}^{2}\right)$ and $E\left(w_{i s j} w_{i t j} w_{i u j} w_{i v j}\right)=0$ for $s \neq t, u, v$.

Note that any of the assumptions, (A-i) to (A-iii), implies (A-v). That is (A-v) is milder than any of (A-i) to (A-iii). We assume the following conditions for $\Sigma_{i} \mathrm{~S}$ :

(A-vi) $\frac{\lambda_{i 1}}{\operatorname{tr}\left(\boldsymbol{\Sigma}_{i}^{2}\right)^{1 / 2}} \rightarrow 0$ as $p \rightarrow \infty$ for $i=1, \ldots, k$.

We recall that $\lambda_{i 1}(i=1, \ldots, k)$ is the largest eigenvalue of $\boldsymbol{\Sigma}_{i}$. Note that $\left\{\lambda_{i 1} / \operatorname{tr}\left(\boldsymbol{\Sigma}_{i}^{2}\right)^{1 / 2}\right\}^{4} \leq \operatorname{tr}\left(\boldsymbol{\Sigma}_{i}^{4}\right) / \operatorname{tr}\left(\boldsymbol{\Sigma}_{i}^{2}\right)^{2}$. We also note that $\liminf _{p \rightarrow \infty} \operatorname{tr}\left(\boldsymbol{\Sigma}_{i}^{2}\right) / p>$ 0 under the assumption that $\liminf _{p \rightarrow \infty} \lambda_{i p}>0$. Thus (A-iv) implies (A-vi). That is (A-vi) is milder than (A-iv). Under (A-v) and (A-vi), we have the following result.

Theorem 4 Assume (A-v) and (A-vi). Then, it holds as $p \rightarrow \infty$ and $n_{i} \rightarrow \infty$, $i=1, \ldots, k$, that

$$
\frac{\left\|\boldsymbol{T}_{\mathbf{n}}-\boldsymbol{\mu}\right\|^{2}-\widehat{\Sigma}_{\mathbf{n}}}{K^{1 / 2}} \Rightarrow N(0,1) .
$$

We assume the following extra condition:

$$
\frac{\sum_{i=1}^{k} \boldsymbol{\mu}^{T} \boldsymbol{\Sigma}_{i} \boldsymbol{\mu} / n_{i}}{K}=o(1)
$$

Note that the condition that $n_{i} \boldsymbol{\mu}^{T} \boldsymbol{\Sigma}_{i} \boldsymbol{\mu} / \operatorname{tr}\left(\boldsymbol{\Sigma}_{i}^{2}\right)=o(1), i=1, \ldots, k$, in Theorem 3 implies (A-vii). Then, we have the following result.

Theorem 5 Assume (A-v) to (A-vii). Then, it holds as $p \rightarrow \infty$ and $n_{i} \rightarrow \infty$, $i=1, \ldots, k$, that

$$
\frac{\widehat{T}_{\mathbf{n}}-\|\boldsymbol{\mu}\|^{2}}{K_{*}^{1 / 2}} \Rightarrow N(0,1) .
$$

When (A-vii) is not met, we have the following result.

Corollary 2 Assume (A-vi) and

(A-viii) $\liminf \frac{\sum_{i=1}^{k} \boldsymbol{\mu}^{T} \boldsymbol{\Sigma}_{i} \boldsymbol{\mu} / n_{i}}{K}>0$ as $p \rightarrow \infty$ and $n_{i} \rightarrow \infty, i=1, \ldots, k$.

Then, it holds as $p \rightarrow \infty$ and $n_{i} \rightarrow \infty, i=1, \ldots, k$, that

$$
\frac{\widehat{T}_{\mathbf{n}}}{\|\boldsymbol{\mu}\|^{2}}=1+o_{p}(1) \text {. }
$$


We consider the following conditions instead of $(\mathrm{A}-\mathrm{v})$ :

$\left(\mathrm{A}-\mathrm{v}^{\prime}\right) \quad \operatorname{Var}_{\boldsymbol{\theta}}\left[\left\{\left(\boldsymbol{x}_{i l}-\boldsymbol{\mu}_{i}\right)^{T}\left(\boldsymbol{x}_{j l^{\prime}}-\boldsymbol{\mu}_{j}\right)\right\}^{2}\right]=O\left\{\operatorname{tr}\left(\boldsymbol{\Sigma}_{i} \boldsymbol{\Sigma}_{j}\right)^{2}\right\}$ and $\operatorname{Var}_{\boldsymbol{\theta}}\left\{\left(\boldsymbol{x}_{i l}-\right.\right.$ $\left.\left.\boldsymbol{\mu}_{i}\right)^{T} \boldsymbol{\Sigma}_{j}\left(\boldsymbol{x}_{i l}-\boldsymbol{\mu}_{i}\right)\right\}=O\left\{\operatorname{tr}\left(\boldsymbol{\Sigma}_{i} \boldsymbol{\Sigma}_{j} \boldsymbol{\Sigma}_{i} \boldsymbol{\Sigma}_{j}\right)\right\}$ as $p \rightarrow \infty$ for $i, j=1, \ldots, k$.

Then, we have the following result.

Corollary 3 After replacing (A-v) with $\left(A-v^{\prime}\right)$, the results in Theorems 4 and 5 are still justified.

Remark 1 From (14) and (16) in Appendix, we note that (A-v) implies (A-v').

Since $\boldsymbol{\Sigma}_{i}$ s are unknown, it is necessary to estimate $K$ (or $K_{*}$ ). Let us consider an estimator of $K$ by

$$
\widehat{K}=2 \sum_{i=1}^{k} \frac{b_{i}^{4} W_{i n_{i}}}{n_{i}\left(n_{i}-1\right)}+4 \sum_{i<j} \frac{b_{i}^{2} b_{j}^{2} \operatorname{tr}\left(\boldsymbol{S}_{i n_{i}} \boldsymbol{S}_{j n_{j}}\right)}{n_{i} n_{j}}
$$

where $W_{i n_{i}}$ is defined by (4). From Lemma 3 in Appendix, we note that $\widehat{K} / K=1+o_{p}(1)$ as $p \rightarrow \infty$ and $n_{i} \rightarrow \infty, i=1, \ldots, k$, under (A-v). Then, we have the following result.

Corollary 4 Assume $(A-v)$ and (A-vi). Then, it holds as $p \rightarrow \infty$ and $n_{i} \rightarrow$ $\infty, i=1, \ldots, k$, that

$$
\frac{\left\|\boldsymbol{T}_{\mathbf{n}}-\boldsymbol{\mu}\right\|^{2}-\widehat{\Sigma}_{\mathbf{n}}}{\widehat{K}^{1 / 2}} \Rightarrow N(0,1) .
$$

Note that $K_{*} / K \rightarrow 1$ as $p \rightarrow \infty$ and $n_{i} \rightarrow \infty, i=1, \ldots, k$, under (A-vii). Then, we have the following result.

Corollary 5 Assume (A-v) to (A-vii). Then, it holds as $p \rightarrow \infty$ and $n_{i} \rightarrow \infty$, $i=1, \ldots, k$, that

$$
\frac{\widehat{T}_{\mathbf{n}}-\|\boldsymbol{\mu}\|^{2}}{\widehat{K}^{1 / 2}} \Rightarrow N(0,1) .
$$

Remark 2 After replacing (A-v) with (A-v'), the results in Corollaries 4 and 5 are still justified.

Let us observe Corollaries 4 and 5 . We set $k=2, b_{1}=1, b_{2}=-1$, $n_{1}=n_{2}=20, \boldsymbol{\Sigma}_{1}=\boldsymbol{B}\left(0.3^{|i-j|^{1 / 3}}\right) \boldsymbol{B}$ and $\boldsymbol{\Sigma}_{2}=\boldsymbol{B}\left(0.4^{|i-j|^{1 / 3}}\right) \boldsymbol{B}$, where

$$
\boldsymbol{B}=\operatorname{diag}\left[\{0.5+1 /(p+1)\}^{1 / 2}, \ldots,\{0.5+p /(p+1)\}^{1 / 2}\right] .
$$

Note that $\operatorname{tr}\left(\boldsymbol{\Sigma}_{i}\right)=p(i=1,2)$. Also, note that $\boldsymbol{\Sigma}_{i}, i=1,2$, hold (A-vi). We considered two cases: (i) $\boldsymbol{\mu}_{1}=\boldsymbol{\mu}_{2}$, and (ii) $\boldsymbol{\mu}_{1}=\left((3 / p)^{1 / 2}, \ldots,(3 / p)^{1 / 2}\right)^{T}$ and $\boldsymbol{\mu}_{2}=\mathbf{0}$ (i.e., $\|\boldsymbol{\mu}\|^{2}=\left\|\boldsymbol{\mu}_{1}-\boldsymbol{\mu}_{2}\right\|^{2}=3$ ). Let $\widetilde{T}=\left(\widehat{T}_{\mathbf{n}}-\|\boldsymbol{\mu}\|^{2}\right) / \widehat{K}^{1 / 2}$. Note that $\widetilde{T}=\left(\left\|\boldsymbol{T}_{\mathbf{n}}-\boldsymbol{\mu}\right\|^{2}-\widehat{\Sigma}_{\mathbf{n}}\right) / \widehat{K}^{1 / 2}$ for case (i). We considered the cases of $p=10,100$ and 1000. We generated $\boldsymbol{x}_{i j}-\boldsymbol{\mu}_{i}, j=1,2, \ldots, \quad(i=1,2)$ independently from a $p$-variate distribution. We considered three distributions: (a) $N_{p}\left(\mathbf{0}, \boldsymbol{\Sigma}_{i}\right)$, (b) $p$-variate $t$-distribution, $t_{p}\left(\mathbf{0}, \boldsymbol{\Sigma}_{i}, \nu\right)$ with mean zero, covariance matrix $\boldsymbol{\Sigma}_{i}$ and 
degrees of freedom $\nu=10$, and (c) $\boldsymbol{\Gamma}_{i} \boldsymbol{w}_{i j}$, where $\boldsymbol{w}_{i j}=\left(w_{i 1 j}, \ldots, w_{i r_{i} j}\right)^{T}$ and $w_{i i^{\prime} j}=\left(y_{i i^{\prime} j}-1\right) / \sqrt{2}\left(i^{\prime}=1, \ldots, r_{i}\right)$ in which $y_{i i^{\prime} j}$ s are i.i.d. as the chi-squared distribution with 1 degree of freedom. Here, $r_{i}=2 p$ and $\boldsymbol{\Gamma}_{i}$ is a $p \times r_{i}$ matrix such that $\boldsymbol{\Gamma}_{i}=\left[\boldsymbol{H}_{i} \boldsymbol{\Lambda}_{i}^{1 / 2} / \sqrt{2},-\boldsymbol{H}_{i} \boldsymbol{\Lambda}_{i}^{1 / 2} / \sqrt{2}\right]$. Note that $\boldsymbol{\Gamma}_{i} \boldsymbol{\Gamma}_{i}^{T}=\boldsymbol{\Sigma}_{i}$. It should be noted that $(\mathrm{A}-\mathrm{v})$ or $\left(\mathrm{A}-\mathrm{v}^{\prime}\right)$ is met in (b) when $\nu$ is sufficiently large. Also, $(\mathrm{A}-\mathrm{v})$ is met in $(\mathrm{c})$.

Independent pseudorandom 2000 observations of $\widetilde{T}$ were generated from each distribution. Let $\widetilde{T}_{r}$ be the $r$ th observation of $\widetilde{T}$ for $r=1, \ldots, 2000$. In the end of the $r$ th replication, we checked whether the inequality, $\widetilde{T}_{r} \leq z_{0.05}$, is true (or false) and defined $P_{i r}=1$ (or 0 ), where $z_{0.05}$ is the upper 0.05 point of $N(0,1)$. We calculated $\bar{P}(0.95)=\sum_{r=1}^{2000} P_{r} / 2000$ as an estimate of $P_{\boldsymbol{\theta}}\left(\widetilde{T} \leq z_{0.05}\right)$. Note that the standard deviation of the estimates is less than 0.011 . From Corollaries 4 and 5 , it holds that $P_{\boldsymbol{\theta}}\left(\widetilde{T} \leq z_{0.05}\right) \rightarrow 0.95$. In Figs. 1 and 2, we gave the histograms of $\widetilde{T}$ together with $\bar{P}(0.95)$ for (a), (b) and (c) when $p=10,100$ and 1000. We considered case (i) in Fig. 1 and case (ii) in Fig. 2. From Corollaries 4 and 5, we also displayed the asymptotic probability density of $\widetilde{T}, N(0,1)$. We observed from Fig. 1 that the histograms become close to the probability density and $\bar{P}(0.95)$ s become close to 0.95 as $p$ increases in case (i). Compared to (i), in view of Fig. 2, the convergence of those quantities seemed to be slow in case (ii) when $p$ is not large enough to meet $K_{*} / K \rightarrow 1$. However, for sufficiently large $p$, we observed that they give adequate performances even in non-Gaussian cases such as (b) and (c).

\section{Estimation of $\operatorname{tr}\left(\Sigma^{2}\right)$}

Throughout this section, we omit the subscript with regard to the population. Yata and Aoshima (2013) developed the extended cross-data-matrix (ECDM) methodology that is an extension of the CDM methodology created by Yata and Aoshima (2010). The ECDM methodology can be applied to obtaining an unbiased estimator of $\operatorname{tr}\left(\boldsymbol{\Sigma}^{2}\right)$ as follows: We assume $n \geq 4$. Let $n_{(1)}=\lceil n / 2\rceil$ and $n_{(2)}=n-n_{(1)}$, where $\lceil x\rceil$ denotes the smallest integer $\geq x$. Let

$$
\begin{aligned}
& \boldsymbol{V}_{n(1)(k)}= \begin{cases}\left\{\lfloor k / 2\rfloor-n_{(1)}+1, \ldots,\lfloor k / 2\rfloor\right\} & \text { if }\lfloor k / 2\rfloor \geq n_{(1)}, \\
\{1, \ldots,\lfloor k / 2\rfloor\} \cup\left\{\lfloor k / 2\rfloor+n_{(2)}+1, \ldots, n\right\} & \text { otherwise; }\end{cases} \\
& \boldsymbol{V}_{n(2)(k)}= \begin{cases}\left\{\lfloor k / 2\rfloor+1, \ldots,\lfloor k / 2\rfloor+n_{(2)}\right\} & \text { if }\lfloor k / 2\rfloor \leq n_{(1)}, \\
\left\{1, \ldots,\lfloor k / 2\rfloor-n_{(1)}\right\} \cup\{\lfloor k / 2\rfloor+1, \ldots, n\} & \text { otherwise }\end{cases}
\end{aligned}
$$

for $k=3, \ldots, 2 n-1$, where $\lfloor x\rfloor$ denotes the largest integer $\leq x$. Let $\#(\boldsymbol{S})$ denote the number of elements in a set $\boldsymbol{S}$. Note that $\#\left(\boldsymbol{V}_{n(l)(k)}\right)=n_{(l)}, l=1,2$, $\boldsymbol{V}_{n(1)(k)} \cap \boldsymbol{V}_{n(2)(k)}=\emptyset$ and $\boldsymbol{V}_{n(1)(k)} \cup \boldsymbol{V}_{n(2)(k)}=\{1, \ldots, n\}$ for $k=3, \ldots, 2 n-1$. Also, note that $i \in \boldsymbol{V}_{n(1)(i+j)}$ and $j \in \boldsymbol{V}_{n(2)(i+j)}$ for $i<j(\leq n)$. Let

$$
\overline{\boldsymbol{x}}_{n(1)(k)}=n_{(1)}^{-1} \sum_{j \in \boldsymbol{V}_{n(1)(k)}} \boldsymbol{x}_{j} \text { and } \quad \overline{\boldsymbol{x}}_{n(2)(k)}=n_{(2)}^{-1} \sum_{j \in \boldsymbol{V}_{n(2)(k)}} \boldsymbol{x}_{j}
$$



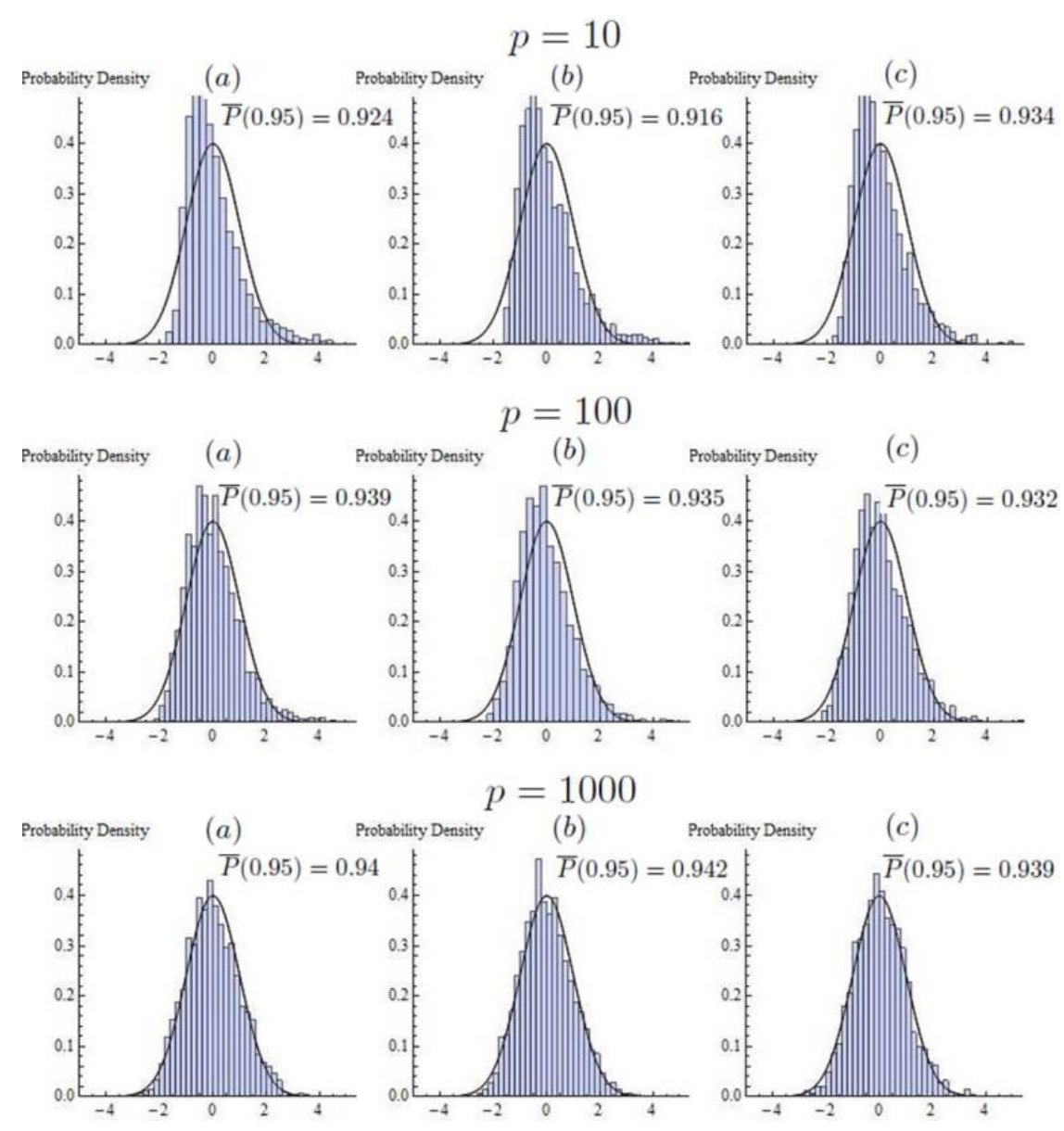

Fig. 1 The histograms of $\widetilde{T}$ together with $\bar{P}(0.95)$ and the probability density of $N(0,1)$ for (a), (b) and (c) when $p=10,100$ and 1000 in case of (i).

for $k=3, \ldots, 2 n-1$. Then, Yata and Aoshima (2013) gave an estimator of $\operatorname{tr}\left(\boldsymbol{\Sigma}^{2}\right)$ by

$$
W_{n}=\frac{2 u_{n}}{n(n-1)} \sum_{i<j}^{n}\left\{\left(\boldsymbol{x}_{i}-\overline{\boldsymbol{x}}_{n(1)(i+j)}\right)^{T}\left(\boldsymbol{x}_{j}-\overline{\boldsymbol{x}}_{n(2)(i+j)}\right)\right\}^{2},
$$

where $u_{n}=n_{(1)} n_{(2)} /\left\{\left(n_{(1)}-1\right)\left(n_{(2)}-1\right)\right\}$. Note that $E_{\boldsymbol{\theta}}\left(W_{n}\right)=\operatorname{tr}\left(\boldsymbol{\Sigma}^{2}\right)$. Aoshima and Yata (2013) and Yata and Aoshima (2013) showed that

$$
\operatorname{Var}_{\boldsymbol{\theta}}\left(\frac{W_{n}}{\operatorname{tr}\left(\boldsymbol{\Sigma}^{2}\right)}\right)=\frac{4}{n^{2}}\{1+o(1)\}+O\left\{\frac{\operatorname{tr}\left(\boldsymbol{\Sigma}^{4}\right)}{\operatorname{tr}\left(\boldsymbol{\Sigma}^{2}\right)^{2} n}\right\} \rightarrow 0
$$




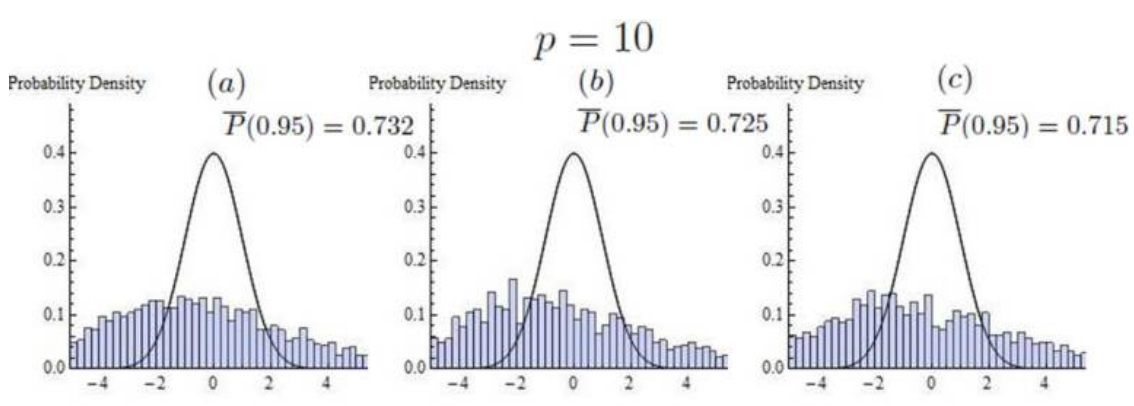

$$
p=100
$$
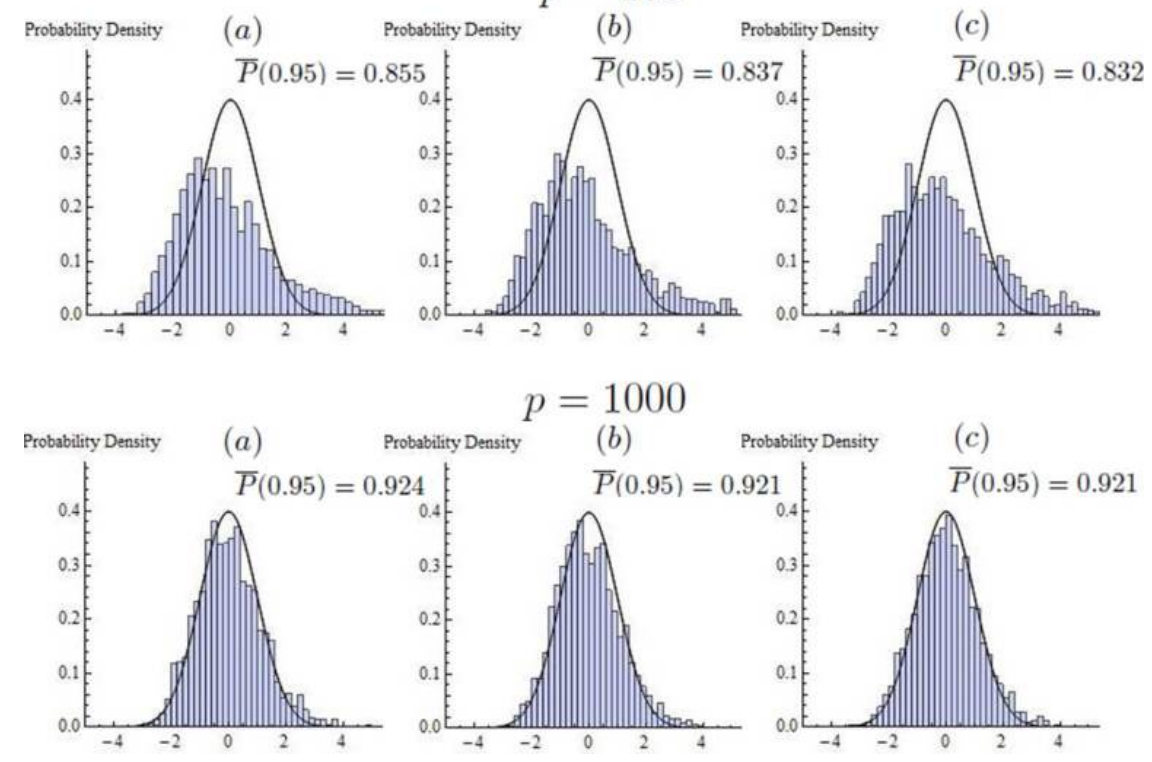

Fig. 2 The histograms of $\widetilde{T}$ together with $\bar{P}(0.95)$ and the probability density of $N(0,1)$ for (a), (b) and (c) when $p=10,100$ and 1000 in case of (ii).

as $p \rightarrow \infty$ and $n \rightarrow \infty$ under (A-v). On the other hand, under (A-v'), we can claim as $p \rightarrow \infty$ and $n \rightarrow \infty$ that

$$
\operatorname{Var}_{\boldsymbol{\theta}}\left(\frac{W_{n}}{\operatorname{tr}\left(\boldsymbol{\Sigma}^{2}\right)}\right)=O\left(n^{-2}\right)+O\left\{\frac{\operatorname{tr}\left(\boldsymbol{\Sigma}^{4}\right)}{\operatorname{tr}\left(\boldsymbol{\Sigma}^{2}\right)^{2} n}\right\} \rightarrow 0 .
$$

Remark 3 Assume (A-i). It holds as $p \rightarrow \infty$ and $n \rightarrow \infty$ that

$$
\operatorname{Var}_{\boldsymbol{\theta}}\left(\frac{W_{n}}{\operatorname{tr}\left(\boldsymbol{\Sigma}^{2}\right)}\right)=\frac{4}{n^{2}}\{1+o(1)\}+8 \frac{\operatorname{tr}\left(\boldsymbol{\Sigma}^{4}\right)}{\operatorname{tr}\left(\boldsymbol{\Sigma}^{2}\right)^{2} n}\{1+o(1)\} .
$$

On the other hand, Bai and Saranadasa (1996) and Srivastava (2005) considered an estimator of $\operatorname{tr}\left(\boldsymbol{\Sigma}^{2}\right)$ by $V_{n}=c_{n}^{-1}\left\{\operatorname{tr}\left(\boldsymbol{S}_{n}^{2}\right)-\operatorname{tr}\left(\boldsymbol{S}_{n}\right)^{2} /(n-1)\right\}$ with $c_{n}=(n-2)(n+1) /(n-1)^{2}$. They showed that, when the population distribution is Gaussian, it holds that $E_{\boldsymbol{\theta}}\left(V_{n}\right)=\operatorname{tr}\left(\boldsymbol{\Sigma}^{2}\right)$ together with (6). It should be 
noted that $V_{n}$ is not an unbiased estimator unless the population distribution is Gaussian. In addition, one cannot claim $\operatorname{Var}_{\boldsymbol{\theta}}\left\{V_{n} / \operatorname{tr}\left(\boldsymbol{\Sigma}^{2}\right)\right\}<\infty$ unless the eighth moments of each variable in $\boldsymbol{z}_{j}$ are uniformly bounded.

\section{Applications}

In this section, we give several applications of the results in Section 2.

\subsection{Confidence regions for $\boldsymbol{\mu}$}

Let $z_{\alpha}$ be a constant such that $P\left\{N(0,1)>z_{\alpha}\right\}=\alpha$. We construct confidence regions for $\boldsymbol{\mu}$ by

$$
\begin{aligned}
& \boldsymbol{R}=\left\{\boldsymbol{\mu} \in R^{p}: \max \left\{\widehat{\Sigma}_{\mathbf{n}}-z_{\alpha / 2} \widehat{K}^{1 / 2}, 0\right\} \leq\left\|\boldsymbol{T}_{\mathbf{n}}-\boldsymbol{\mu}\right\|^{2} \leq \widehat{\Sigma}_{\mathbf{n}}+z_{\alpha / 2} \widehat{K}^{1 / 2}\right\} ; \\
& \boldsymbol{R}_{U}=\left\{\boldsymbol{\mu} \in R^{p}:\left\|\boldsymbol{T}_{\mathbf{n}}-\boldsymbol{\mu}\right\|^{2} \leq \widehat{\Sigma}_{\mathbf{n}}+z_{\alpha} \widehat{K}^{1 / 2}\right\},
\end{aligned}
$$

where $\alpha \in(0,1 / 2)$. Then, from Corollary 4 , it holds as $p \rightarrow \infty$ and $n_{i} \rightarrow \infty$, $i=1, \ldots, k$, that

$$
P_{\boldsymbol{\theta}}(\boldsymbol{\mu} \in \boldsymbol{R})=1-\alpha+o(1) \quad \text { and } \quad P_{\boldsymbol{\theta}}\left(\boldsymbol{\mu} \in \boldsymbol{R}_{U}\right)=1-\alpha+o(1)
$$

under $(\mathrm{A}-\mathrm{v})$ and $(\mathrm{A}-\mathrm{vi})$.

Remark 4 When $\widehat{\Sigma}_{\mathbf{n}}>z_{\alpha / 2} \widehat{K}^{1 / 2}, \boldsymbol{R}$ indicates that $\boldsymbol{\mu}$ is included in the region sandwiched by the two $p$-dimensional spheres with radii of $\left(\widehat{\Sigma}_{\mathbf{n}}-z_{\alpha / 2} \widehat{K}^{1 / 2}\right)^{1 / 2}$ and $\left(\widehat{\Sigma}_{\mathbf{n}}+z_{\alpha / 2} \widehat{K}^{1 / 2}\right)^{1 / 2}$ from center $\boldsymbol{T}_{\mathbf{n}}$. See Section 2 in Aoshima and Yata (2011a) for the details.

4.2 Confidence intervals for $\|\boldsymbol{\mu}\|^{2}$

We construct confidence intervals for $\|\boldsymbol{\mu}\|^{2}$ by

$$
\begin{aligned}
& I=\left[\max \left\{\widehat{T}_{\mathbf{n}}-z_{\alpha / 2} \widehat{K}^{1 / 2}, 0\right\}, \widehat{T}_{\mathbf{n}}+z_{\alpha / 2} \widehat{K}^{1 / 2}\right] ; \\
& I_{L}=\left[\max \left\{\widehat{T}_{\mathbf{n}}-z_{\alpha} \widehat{K}^{1 / 2}, 0\right\}, \infty\right),
\end{aligned}
$$

where $\alpha \in(0,1 / 2)$. Then, from Corollary 5 , it holds as $p \rightarrow \infty$ and $n_{i} \rightarrow \infty$, $i=1, \ldots, k$, that

$$
P_{\boldsymbol{\theta}}\left(\|\boldsymbol{\mu}\|^{2} \in I\right)=1-\alpha+o(1) \quad \text { and } \quad P_{\boldsymbol{\theta}}\left(\|\boldsymbol{\mu}\|^{2} \in I_{L}\right)=1-\alpha+o(1)
$$

under (A-v) to (A-vii). We emphasize that one can apply $I$ and $I_{L}$ to the discriminant analysis for high-dimensional data. Refer to Section 4 in Aoshima and Yata (2011a, 2011c) and Sections 3 and 4 in Aoshima and Yata (2013). 
4.3 Test of $\boldsymbol{\mu}=\mathbf{0}$ against $\boldsymbol{\mu} \neq \mathbf{0}$

We consider the following test:

$$
H_{0}: \boldsymbol{\mu}=\mathbf{0} \quad \text { vs. } \quad H_{1}: \boldsymbol{\mu} \neq \mathbf{0} \text {. }
$$

For given $\alpha \in(0,1 / 2)$, we test the hypothesis ( 7$)$ by

$$
\text { rejecting } H_{0} \Longleftrightarrow \frac{\widehat{T}_{\mathbf{n}}}{\widehat{K}^{1 / 2}}>z_{\alpha} \text {. }
$$

Then, from Corollary 5 , it holds as $p \rightarrow \infty$ and $n_{i} \rightarrow \infty, i=1, \ldots, k$, that

$$
\text { size }=\alpha+o(1) \quad \text { and } \quad \text { power }=\Phi\left(\frac{\|\boldsymbol{\mu}\|^{2}}{K^{1 / 2}}-z_{\alpha}\right)+o(1)
$$

under (A-v) to (A-vii), where $\Phi(\cdot)$ denotes the c.d.f. of $N(0,1)$. If one cannot assume (A-vii) in case of $\boldsymbol{\mu} \neq \mathbf{0}$, from Corollaries 2 and 5 , it holds as $p \rightarrow \infty$ and $n_{i} \rightarrow \infty, i=1, \ldots, k$, that

$$
\text { size }=\alpha+o(1) \text { and } \text { power }=1+o(1)
$$

under (A-v), (A-vi) and (A-viii).

Remark 5 When $k=2$ and $\left(b_{1}, b_{2}\right)=(1,-1)$, Chen and Qin (2010) also gave (8) under slightly different conditions.

Remark 6 After replacing $(\mathrm{A}-\mathrm{v})$ with $\left(\mathrm{A}-\mathrm{v}^{\prime}\right)$, all the results in Section 4 are still justified.

\section{Sample size determination}

In this section, we provide sample size determination so as to satisfy prespecified accuracy on inference.

5.1 Given-bandwidth confidence region for $\boldsymbol{\mu}$

We consider constructing a given-bandwidth confidence region for $\boldsymbol{\mu}$ by

$$
\boldsymbol{R}_{\mathbf{n}}=\left\{\boldsymbol{\mu} \in R^{p}: \max \left\{\widehat{\Sigma}_{\mathbf{n}}-\delta, 0\right\} \leq\left\|\boldsymbol{T}_{\mathbf{n}}-\boldsymbol{\mu}\right\|^{2} \leq \widehat{\Sigma}_{\mathbf{n}}+\delta\right\}
$$

for given $\delta(>0)$. We assume $\delta=o\left\{\min _{i=1, \ldots, k} \operatorname{tr}\left(\boldsymbol{\Sigma}_{i}^{2}\right)^{1 / 2}\right\}$. For given $\alpha \in$ $(0,1 / 2)$, we determine the sample size so as to satisfy

$$
P_{\boldsymbol{\theta}}\left(\boldsymbol{\mu} \in \boldsymbol{R}_{\mathbf{n}}\right) \geq 1-\alpha
$$


Aoshima and Yata (2011a) considered sample size determination as follows: From the fact that $\operatorname{tr}\left(\boldsymbol{\Sigma}_{i} \boldsymbol{\Sigma}_{j}\right) \leq\left\{\operatorname{tr}\left(\boldsymbol{\Sigma}_{i}^{2}\right) \operatorname{tr}\left(\boldsymbol{\Sigma}_{j}^{2}\right)\right\}^{1 / 2}$, it holds $K^{1 / 2} \leq \sqrt{2} \sum_{i=1}^{k} b_{i}^{2}$ $\operatorname{tr}\left(\boldsymbol{\Sigma}_{i}^{2}\right)^{1 / 2} /\left(n_{i}-1\right)$. One may choose $n_{i}$ s such that

$$
\min \sum_{i=1}^{k} n_{i} \quad \text { subject to } \sqrt{2} \sum_{i=1}^{k} b_{i}^{2} \operatorname{tr}\left(\boldsymbol{\Sigma}_{i}^{2}\right)^{1 / 2} /\left(n_{i}-1\right) \leq \delta / z_{\alpha / 2} .
$$

Then, the sample size is determined by

$$
n_{i} \geq \frac{z_{\alpha / 2} \sqrt{2}}{\delta}\left|b_{i}\right| \operatorname{tr}\left(\Sigma_{i}^{2}\right)^{1 / 4} \sum_{j=1}^{k}\left|b_{j}\right| \operatorname{tr}\left(\Sigma_{j}^{2}\right)^{1 / 4}+1 \quad\left(=C_{i}, \text { say }\right)
$$

for each $\pi_{i}$. Note that $n_{i} \rightarrow \infty, i=1, \ldots, k$, as $p \rightarrow \infty$.

Theorem 6 (Aoshima and Yata, 2011a) Assume (A-iv) and either (A-ii) or (A-iii). Then, for $n_{i}$ s satisfying (10), it holds as $p \rightarrow \infty$ that

$$
\liminf P_{\boldsymbol{\theta}}\left(\boldsymbol{\mu} \in \boldsymbol{R}_{\mathbf{n}}\right) \geq 1-\alpha .
$$

We can claim (11) under mild conditions as follows.

Theorem 7 Assume (A-v) and (A-vi). Then, for $n_{i} s$ satisfying (10), (11) holds as $p \rightarrow \infty$.

\subsection{Two-stage procedure}

Since $\boldsymbol{\Sigma}_{i}$ s are unknown, it is necessary to estimate $C_{i}$ in (10). We proceed with the following two steps along the lines of the elastic two-stage procedure given by Aoshima and Yata (2011b):

1. Choose $m_{i}(\geq 4), i=1, \ldots, k$, such as

$$
\frac{m_{i}}{C_{i}} \leq 1, \quad \frac{C_{i}}{m_{i}^{2}} \rightarrow 0 \text { and } \frac{C_{i}}{m_{i}} \frac{\operatorname{tr}\left(\boldsymbol{\Sigma}_{i}^{4}\right)}{\operatorname{tr}\left(\boldsymbol{\Sigma}_{i}^{2}\right)^{2}} \rightarrow 0 \text { as } p \rightarrow \infty \text { under (A-vi). }
$$

Note that (12) is met when $\liminf _{p \rightarrow \infty} m_{i} / C_{i}>0$ and $\lim \sup _{p \rightarrow \infty} m_{i} / C_{i}<1$. Take pilot samples, $\boldsymbol{x}_{i j}, j=1, \ldots, m_{i}$, of size $m_{i}$ from each $\pi_{i}$. Then, calculate $W_{i m_{i}}$ according to (4). Define the total sample size for each $\pi_{i}$ by

$$
N_{i}=\max \left\{m_{i}, \quad\left\lceil\frac{z_{\alpha / 2} \sqrt{2}}{\delta}\left|b_{i}\right| W_{i m_{i}}^{1 / 4} \sum_{j=1}^{k}\left|b_{j}\right| W_{j m_{j}}^{1 / 4}\right\rceil+1\right\},
$$

where $\lceil x\rceil$ denotes the smallest integer $\geq x$.

2. For each $i$, if $N_{i}=m_{i}$, do not take any additional samples from $\pi_{i}$ and otherwise, that is if $N_{i}>m_{i}$, take additional samples, $\boldsymbol{x}_{i j}, j=m+1, \ldots, N_{i}$, of size $N_{i}-m_{i}$ from $\pi_{i}$. By combining the initial samples and the additional samples, calculate $\overline{\boldsymbol{x}}_{i N_{i}}$ and $\boldsymbol{S}_{i N_{i}}, i=1, \ldots, k$. Let $\boldsymbol{N}=\left(N_{1}, \ldots, N_{k}\right)$. Then, define $\boldsymbol{R}_{\mathbf{N}}$ according to (9) with $\boldsymbol{T}_{\mathbf{N}}=\sum_{i=1}^{k} b_{i} \overline{\boldsymbol{x}}_{i N_{i}}$ and $\widehat{\Sigma}_{\mathbf{N}}=\sum_{i=1}^{k} b_{i}^{2} \operatorname{tr}\left(\boldsymbol{S}_{i N_{i}}\right) / N_{i}$.

We have the following theorem. 
Theorem 8 Assume $(A-v)$ and (A-vi). For two-stage procedure given by (12) and (13), we have as $p \rightarrow \infty$ that

$$
\liminf P_{\boldsymbol{\theta}}\left(\boldsymbol{\mu} \in \boldsymbol{R}_{\mathbf{N}}\right) \geq 1-\alpha
$$

Remark 7 The results in Theorems 7 and 8 are still justified under (A-v') instead of $(\mathrm{A}-\mathrm{v})$.

Remark 8 Under (12), (A-v) and (A-vi), it holds as $p \rightarrow \infty$ that $\operatorname{Var}_{\boldsymbol{\theta}}\left\{W_{i m_{i}}\right.$ $\left.\operatorname{tr}\left(\boldsymbol{\Sigma}_{i}^{2}\right)\right\}=o\left(C_{i}^{-1}\right)$. Then, we can claim as $p \rightarrow \infty$ that $N_{i} / C_{i}=1+o_{p}(1)$, which is in the HDLSS situation in the sense that $N_{i} / p=o_{p}(1)$ under the condition that $\max _{i=1, \ldots, k} \operatorname{tr}\left(\boldsymbol{\Sigma}_{i}^{2}\right)^{1 / 2} / \delta=o(p)$

Remark 9 One may choose $m_{i}(\geq 4)$ such as $m_{i} / C_{i}>1$ for some $i$. Then, the assertion in Theorem 8 can still be claimed. However, it may cause oversampling in the sense that $N_{i} / C_{i}>1$ w.p.1.

Remark 10 One can obtain sample size determination both for the confidence interval (Section 4.2) and for the test (Section 4.3) as well in similar fashion. Under $(\mathrm{A}-\mathrm{v})$ and $(\mathrm{A}-\mathrm{vi})$, it can be verified to ensure the accuracy required in Yata and Aoshima (2012, Sections 2 and 3) and Aoshima and Yata (2011a, Section 3). We omit the details for brevity.

\subsection{Simulation}

In order to study the performance of the two-stage procedure given by (12) and (13), we used computer simulations. Our goal was to construct a $95 \%$ givenbandwidth confidence region, $\boldsymbol{R}_{\mathbf{N}}$. In other words, we set $\alpha=0.05$. We set $\boldsymbol{\mu}_{i^{\prime}}=(0, \ldots, 0)^{T}, b_{i^{\prime}}=(-1)^{i^{\prime}-1}$ and $\boldsymbol{\Sigma}_{i^{\prime}}=c_{i^{\prime}} \boldsymbol{B}\left(\rho_{i^{\prime}}^{|i-j|^{1 / 3}}\right) \boldsymbol{B}, i^{\prime}=1, \ldots, k$, where $\boldsymbol{B}$ is defined by $(3)$. Note that $\operatorname{tr}\left(\boldsymbol{\Sigma}_{i}\right)=c_{i} p(i=1, . ., k)$. We considered two cases: (i) $k=2, \delta=5,\left(c_{1}, c_{2}\right)=(1,1),\left(\rho_{1}, \rho_{2}\right)=(0.3,0.4)$ and $\left(m_{1}, m_{2}\right)=$ $(10,10)$, and (ii) $k=4, \delta=10,\left(c_{1}, c_{2}, c_{3}, c_{4}\right)=(1,1,1.2,1.2),\left(\rho_{1}, \rho_{2}, \rho_{3}, \rho_{4}\right)=$ $(0.3,0.4,0.3,0.4)$ and $\left(m_{1}, m_{2}, m_{3}, m_{4}\right)=(10,10,15,15)$. In Table 1 , we generated independent pseudorandom observations from $\pi_{i}: N_{p}\left(\mathbf{0}, \boldsymbol{\Sigma}_{i}\right), i=$ $1, \ldots, k$. In Table 2, we generated them from $\boldsymbol{\Gamma}_{i} \boldsymbol{w}_{i j}$, where $\boldsymbol{\Gamma}_{i}=\boldsymbol{H}_{i} \boldsymbol{\Lambda}_{i}^{1 / 2}$, $\boldsymbol{w}_{i j}=\left(w_{i 1 j}, \ldots, w_{i p j}\right)^{T}$ and $w_{i i^{\prime} j}=y_{i i^{\prime} j}-1\left(i^{\prime}=1, \ldots, p\right)$ in which $y_{i i^{\prime} j}$ s are i.i.d. as the Poisson distribution with parameter $\lambda=1$ for $\pi_{i}, i=1, \ldots, k$.

When $p=200$ and 1000, we used the two-stage procedure given by (12) and (13). The findings were obtained by averaging the outcomes from 2000 $(=R$, say) replications. Under a fixed scenario, suppose that the $r$ th replication ends with $N_{i}=n_{i r}(i=1, \ldots, k)$ observations and the corresponding confidence region with $\boldsymbol{n}_{r}=\left(n_{1 r}, \ldots, n_{k r}\right)$ for $r=1, \ldots, R$. Let $\bar{n}_{i}=R^{-1} \sum_{r=1}^{R} n_{i r}$ and $V\left(n_{i}\right)=(R-1)^{-1} \sum_{r=1}^{R}\left(n_{i r}-\bar{n}_{i}\right)^{2}$. In the end of the $r$ th replication, we checked whether $\boldsymbol{\mu}$ does (or does not) belong to the corresponding confidence region and defined $P_{r}=1$ (or 0) accordingly. Let $\bar{P}=R^{-1} \sum_{r=1}^{R} P_{r}$, which 
Table 1 Required sample size and coverage probability given by (12) and (13) when $\pi_{i}$ : $N_{p}\left(\mathbf{0}, \boldsymbol{\Sigma}_{i}\right), i=1, \ldots, k$.

\begin{tabular}{|c|c|c|c|c|c|c|}
\hline & $C_{i}$ & $\bar{n}_{i}$ & $\bar{n}_{i}-C_{i}$ & $V\left(n_{i}\right)$ & $\bar{P}$ & $s(\bar{P})$ \\
\hline & \multicolumn{6}{|c|}{ Case $(\mathrm{i}): k=2, \delta=5$ and $\left(m_{1}, m_{2}\right)=(10,10)$} \\
\hline & \multicolumn{6}{|c|}{$p=200$} \\
\hline$\pi_{1}$ & 22.67 & 22.67 & 0.0 & 8.42 & \multirow{2}{*}{0.957} & \multirow[t]{2}{*}{0.00456} \\
\hline$\pi_{2}$ & 25.29 & 25.25 & -0.04 & $\begin{array}{c}17.51 \\
p=1000\end{array}$ & & \\
\hline$\pi_{1}$ & 49.87 & 49.55 & -0.31 & 30.86 & \multirow[t]{2}{*}{0.954} & \multirow[t]{2}{*}{0.00468} \\
\hline$\pi_{2}$ & 56.1 & 55.82 & -0.28 & 47.26 & & \\
\hline & \multicolumn{6}{|c|}{ Case (ii): $k=4, \delta=10$ and $\left(m_{1}, m_{2}, m_{3}, m_{4}\right)=(10,10,15,15)$} \\
\hline & \multicolumn{6}{|c|}{$p=200$} \\
\hline$\pi_{1}$ & 23.7 & 23.76 & 0.06 & 6.29 & \multirow[t]{4}{*}{0.958} & \multirow[t]{4}{*}{0.00449} \\
\hline$\pi_{2}$ & 26.45 & 26.26 & -0.19 & 11.32 & & \\
\hline$\pi_{3}$ & 25.87 & 25.96 & 0.09 & 3.89 & & \\
\hline$\pi_{4}$ & 28.88 & 28.88 & 0.0 & $\begin{array}{c}8.82 \\
p=1000\end{array}$ & & \\
\hline$\pi_{1}$ & 52.2 & 51.97 & -0.23 & 21.6 & \multirow[t]{4}{*}{0.95} & \multirow[t]{4}{*}{0.0049} \\
\hline$\pi_{2}$ & 58.73 & 58.26 & -0.47 & 33.03 & & \\
\hline$\pi_{3}$ & 57.09 & 57.07 & -0.02 & 12.55 & & \\
\hline$\pi_{4}$ & 64.24 & 63.96 & -0.28 & 21.95 & & \\
\hline
\end{tabular}

estimates the target coverage probability, having its estimated standard error $s(\bar{P})$ where $s^{2}(\bar{P})=R^{-1} \bar{P}(1-\bar{P})$.

Let us explain, for example, the entries from the second block for case (i) in Table 1 that were given when $p=1000$. We had $C_{1}=49.87$ and $C_{2}=56.1$ from (10). From 2000 independent replications, we observed $\bar{n}_{1}=49.55\left(\bar{n}_{1}-\right.$ $\left.C_{1}=-0.31\right), \bar{n}_{2}=55.82\left(\bar{n}_{2}-C_{2}=-0.28\right)$ and $\bar{P}=0.954$ together with $V\left(n_{1}\right)=30.86, V\left(n_{2}\right)=47.26$ and $s(\bar{P})=0.00468$. Throughout, the twostage procedure seemed to construct required confidence regions successfully even for a discrete case such as in Table 2.

\section{Example}

We analyzed gene expression data given by Chiaretti et al. (2004) in which the data set consists of $12625(=p)$ genes. The expression measures were obtained by using the three-step robust multichip average (RMA) preprocessing method. Refer to Pollard et al. (2005) as well for the details. The data set had two tumor cellular subtypes, B-cell and T-cell. We divided each type into two groups with respect to the relapse as follows: (a) $\pi_{1}$ : B-cell with the relapse ( $n_{1}=50$ samples); (b) $\pi_{2}$ : B-cell without the relapse $\left(n_{2}=26\right.$ samples); (c) $\pi_{3}$ : T-cell with the relapse $\left(n_{3}=15\right.$ samples $)$; and $(\mathrm{d}) \pi_{4}$ : T-cell without the relapse $\left(n_{4}=9\right.$ samples $)$. 
Table 2 Required sample size and coverage probability given by (12) and (13) when $\boldsymbol{\Gamma}_{i}=$ $\boldsymbol{H}_{i} \boldsymbol{\Lambda}_{i}^{1 / 2}, \boldsymbol{w}_{i j}=\left(w_{i 1 j}, \ldots, w_{i p j}\right)^{T}$ and $w_{i i^{\prime} j}=y_{i i^{\prime} j}-1\left(i^{\prime}=1, \ldots, p\right)$ in which $y_{i i^{\prime} j}$ s are i.i.d. as the Poisson distribution with parameter $\lambda=1$ for $\pi_{i} i=1, \ldots, k$.

\begin{tabular}{|c|c|c|c|c|c|c|}
\hline & $C_{i}$ & $\bar{n}_{i}$ & $\bar{n}_{i}-C_{i}$ & $V\left(n_{i}\right)$ & $\bar{P}$ & $s(\bar{P})$ \\
\hline & \multicolumn{6}{|c|}{ Case (i): $k=2, \delta=5$ and $\left(m_{1}, m_{2}\right)=(10,10)$} \\
\hline & \multicolumn{6}{|c|}{$p=200$} \\
\hline$\pi_{1}$ & 22.67 & 22.51 & -0.16 & 10.06 & \multirow[t]{2}{*}{0.958} & \multirow[t]{2}{*}{0.00451} \\
\hline$\pi_{2}$ & 25.29 & 25.06 & -0.23 & $\begin{array}{c}19.89 \\
p=1000\end{array}$ & & \\
\hline$\pi_{1}$ & 49.87 & 49.74 & -0.13 & 32.03 & \multirow[t]{2}{*}{0.955} & \multirow[t]{2}{*}{0.00466} \\
\hline$\pi_{2}$ & 56.1 & 55.89 & -0.21 & 50.49 & & \\
\hline & \multicolumn{6}{|c|}{ Case (ii): $k=4, \delta=10$ and $\left(m_{1}, m_{2}, m_{3}, m_{4}\right)=(10,10,15,15)$} \\
\hline & \multicolumn{6}{|c|}{$p=200$} \\
\hline$\pi_{1}$ & 23.7 & 23.65 & -0.05 & 6.65 & \multirow[t]{4}{*}{0.954} & \multirow[t]{4}{*}{0.00471} \\
\hline$\pi_{2}$ & 26.45 & 26.15 & -0.3 & 14.02 & & \\
\hline$\pi_{3}$ & 25.87 & 25.97 & 0.1 & 5.06 & & \\
\hline$\pi_{4}$ & 28.88 & 28.84 & -0.04 & $\begin{array}{c}11.78 \\
p=1000\end{array}$ & & \\
\hline$\pi_{1}$ & 52.2 & 52.02 & -0.18 & 22.79 & \multirow[t]{4}{*}{0.955} & \multirow[t]{4}{*}{0.00466} \\
\hline$\pi_{2}$ & 58.73 & 58.52 & -0.21 & 40.09 & & \\
\hline$\pi_{3}$ & 57.09 & 57.11 & -0.02 & 13.63 & & \\
\hline$\pi_{4}$ & 64.24 & 64.16 & -0.08 & 24.75 & & \\
\hline
\end{tabular}

6.1 Tests of $\boldsymbol{\mu}_{1}=\boldsymbol{\mu}_{3}$ and $\boldsymbol{\mu}_{2}=\boldsymbol{\mu}_{4}$

We first considered testing $\boldsymbol{\mu}_{1}=\boldsymbol{\mu}_{3}$. We set $\alpha=0.05$. We calculated $\widehat{T}_{\mathbf{n}}=$ $\left\|\overline{\boldsymbol{x}}_{1 n_{1}}-\overline{\boldsymbol{x}}_{3 n_{3}}\right\|^{2}-\operatorname{tr}\left(\boldsymbol{S}_{1 n_{1}}\right) / n_{1}-\operatorname{tr}\left(\boldsymbol{S}_{3 n_{3}}\right) / n_{3}=1267$ and $\widehat{K}=2\left(W_{1 n_{1}} /\left\{n_{1}\left(n_{1}-\right.\right.\right.$ $\left.1)\}+W_{3 n_{3}} /\left\{n_{3}\left(n_{3}-1\right)\right\}\right)+4 \operatorname{tr}\left(\boldsymbol{S}_{1 n_{1}} \boldsymbol{S}_{3 n_{3}}\right) /\left(n_{1} n_{3}\right)=4331$ with $W_{1 n_{1}}=2.84 \times$ $10^{5}, W_{3 n_{3}}=2.83 \times 10^{5}$ and $\operatorname{tr}\left(\boldsymbol{S}_{1 n_{1}} \boldsymbol{S}_{3 n_{3}}\right)=2.64 \times 10^{5}$ according to (4). Hence, we obtained $\widehat{T}_{\mathbf{n}} / \widehat{K}^{1 / 2}=19.3$. From the fact that

$$
\frac{\widehat{T}_{\mathbf{n}}}{\widehat{K}^{1 / 2}}>z_{\alpha}=1.64
$$

we rejected the null hypothesis, $\boldsymbol{\mu}_{1}=\boldsymbol{\mu}_{3}$, with size 0.05 according to Section 4.3. According to Section 4.2, we calculated $95 \%$ confidence intervals for $\|\boldsymbol{\mu}\|^{2}=\left\|\boldsymbol{\mu}_{1}-\boldsymbol{\mu}_{3}\right\|^{2}$ as follows:

$$
\begin{aligned}
& I=[1138,1396] \\
& I_{L}=\left[\max \left\{\widehat{T}_{\mathbf{n}}-z_{\alpha} \widehat{K}^{1 / 2}, 0\right\}, \infty\right)=[1159, \infty) .
\end{aligned}
$$

Since both $I$ and $I_{L}$ did not include $\|\boldsymbol{\mu}\|=0$, we concluded $\boldsymbol{\mu}_{1} \neq \boldsymbol{\mu}_{3}$ significantly. 
Similarly, we considered testing $\boldsymbol{\mu}_{2}=\boldsymbol{\mu}_{4}$. From the fact that

$$
\begin{aligned}
& \frac{\widehat{T}_{\mathbf{n}}}{\widehat{K}^{1 / 2}} \\
& =\frac{\left\|\overline{\boldsymbol{x}}_{2 n_{2}}-\overline{\boldsymbol{x}}_{4 n_{4}}\right\|^{2}-\operatorname{tr}\left(\boldsymbol{S}_{2 n_{2}}\right) / n_{2}-\operatorname{tr}\left(\boldsymbol{S}_{4 n_{4}}\right) / n_{4}}{\sqrt{2 W_{2 n_{2}} /\left\{n_{2}\left(n_{2}-1\right)\right\}+2 W_{4 n_{4}} /\left\{n_{4}\left(n_{4}-1\right)\right\}+4 \operatorname{tr}\left(\boldsymbol{S}_{2 n_{2}} \boldsymbol{S}_{4 n_{4}}\right) /\left(n_{2} n_{4}\right)}} \\
& =23.6>z_{\alpha},
\end{aligned}
$$

we rejected the null hypothesis, $\boldsymbol{\mu}_{2}=\boldsymbol{\mu}_{4}$, with size $\alpha=0.05$.

6.2 Confidence region for $\boldsymbol{\mu}=\left(\boldsymbol{\mu}_{1}-\boldsymbol{\mu}_{3}\right)-\left(\boldsymbol{\mu}_{2}-\boldsymbol{\mu}_{4}\right)$

We considered constructing a confidence region for $\boldsymbol{\mu}=\left(\boldsymbol{\mu}_{1}-\boldsymbol{\mu}_{3}\right)-\left(\boldsymbol{\mu}_{2}-\boldsymbol{\mu}_{4}\right)$. We set $\alpha=0.05$. We calculated $\boldsymbol{T}_{\mathbf{n}}=\left(\overline{\boldsymbol{x}}_{1 n_{1}}-\overline{\boldsymbol{x}}_{3 n_{3}}\right)-\left(\overline{\boldsymbol{x}}_{2 n_{2}}-\overline{\boldsymbol{x}}_{4 n_{4}}\right)=$ $(0.151,-0.193, \ldots, 0.115,-0.102)^{T}, \widehat{K}=2 \sum_{i=1}^{4} W_{i n_{i}} /\left\{n_{i}\left(n_{i}-1\right)\right\}+4 \sum_{i<j}$ $\operatorname{tr}\left(\boldsymbol{S}_{i n_{i}} \boldsymbol{S}_{j n_{j}}\right) /\left(n_{i} n_{j}\right)=20010$ and $\widehat{\Sigma}_{\mathbf{n}}=\sum_{i=1}^{4} \operatorname{tr}\left(\boldsymbol{S}_{i n_{i}}\right) / n_{i}=563$. According to Section 4.1, we obtained a confidence region as

$$
\begin{aligned}
\boldsymbol{R} & =\left\{\boldsymbol{\mu} \in R^{p}: \max \left\{\widehat{\Sigma}_{\mathbf{n}}-z_{\alpha / 2} \widehat{K}^{1 / 2}, 0\right\} \leq\left\|\boldsymbol{T}_{\mathbf{n}}-\boldsymbol{\mu}\right\|^{2} \leq \widehat{\Sigma}_{\mathbf{n}}+z_{\alpha / 2} \widehat{K}^{1 / 2}\right\} \\
& =\left\{\boldsymbol{\mu} \in R^{p}: 285 \leq\left\|\boldsymbol{T}_{\mathbf{n}}-\boldsymbol{\mu}\right\|^{2} \leq 840\right\} .
\end{aligned}
$$

When $\boldsymbol{\mu}=\mathbf{0}$, we had $\left\|\boldsymbol{T}_{\mathbf{n}}-\boldsymbol{\mu}\right\|^{2}=\left\|\boldsymbol{T}_{\mathbf{n}}\right\|^{2}=651$, so that $\boldsymbol{\mu}=\mathbf{0} \in \boldsymbol{R}$. Thus we considered $\mathbf{0}$ as a likely candidate of $\boldsymbol{\mu}=\left(\boldsymbol{\mu}_{1}-\boldsymbol{\mu}_{3}\right)-\left(\boldsymbol{\mu}_{2}-\boldsymbol{\mu}_{4}\right)$. In other words, we concluded that there is not much significant difference between B-cell and T-cell in terms of the relapse.

6.3 Given-bandwidth confidence region for $\boldsymbol{\mu}=\boldsymbol{\mu}_{1}-\boldsymbol{\mu}_{2}$

We considered constructing a given-bandwidth confidence region for $\boldsymbol{\mu}=\boldsymbol{\mu}_{1}-$ $\boldsymbol{\mu}_{2}$ along the line of Section 5.2. We set $\alpha=0.05, \delta=150$ and $m_{1}=m_{2}=15$. By using pilot samples of sizes $m_{1}=m_{2}=15$, we calculated $W_{1 m_{1}}=2.96 \times 10^{5}$ and $W_{2 m_{2}}=2.21 \times 10^{5}$ according to (4). From (13), we calculated the total sample sizes as

$$
N_{1}=\max \left\{15,\left\lceil\frac{z_{\alpha / 2} \sqrt{2}}{\delta} W_{1 m_{1}}^{1 / 4} \sum_{j=1}^{2} W_{j m_{j}}^{1 / 4}\right\rceil+1\right\}=21 \quad \text { and } \quad N_{2}=20 .
$$

So, we took the next 6 samples from $\pi_{1}$ and the next 5 samples from $\pi_{2}$. Then, we had $\boldsymbol{T}_{\mathbf{N}}=\overline{\boldsymbol{x}}_{1 N_{1}}-\overline{\boldsymbol{x}}_{2 N_{2}}=(0.101,-0.185, \ldots, 0.012,0.025)^{T}$ and $\widehat{\Sigma}_{\mathbf{N}}=\operatorname{tr}\left(\boldsymbol{S}_{1 N_{1}}\right) / N_{1}+\operatorname{tr}\left(\boldsymbol{S}_{2 N_{2}}\right) / N_{2}=259$. According to (9), we constructed a given-bandwidth confidence region as

$$
\begin{aligned}
\boldsymbol{R}_{\mathbf{N}} & =\left\{\boldsymbol{\mu} \in R^{p}: \max \left\{\widehat{\Sigma}_{\mathbf{N}}-\delta, 0\right\} \leq\left\|\boldsymbol{T}_{\mathbf{N}}-\boldsymbol{\mu}\right\|^{2} \leq \widehat{\Sigma}_{\mathbf{N}}+\delta\right\} \\
& =\left\{\boldsymbol{\mu} \in R^{p}: 109 \leq\left\|\boldsymbol{T}_{\mathbf{N}}-\boldsymbol{\mu}\right\|^{2} \leq 409\right\} .
\end{aligned}
$$


When $\boldsymbol{\mu}=\mathbf{0}$, we had $\left\|\boldsymbol{T}_{\mathbf{N}}-\boldsymbol{\mu}\right\|^{2}=\left\|\boldsymbol{T}_{\mathbf{N}}\right\|^{2}=243$, so that $\boldsymbol{\mu}=\mathbf{0} \in \boldsymbol{R}_{\mathbf{N}}$. Thus we considered $\mathbf{0}$ as a likely candidate of $\boldsymbol{\mu}=\boldsymbol{\mu}_{1}-\boldsymbol{\mu}_{2}$. If one considers a mean structure such as $\boldsymbol{\mu}=\boldsymbol{\mu}_{1}-\boldsymbol{\mu}_{2}=c(1, \ldots, 1)^{T}$ with an unknown constant $c$, we can provide an allowable range of $c$ by $c \in[-0.126,0.104]$ such that $\boldsymbol{\mu}=c(1, \ldots, 1)^{T} \in \boldsymbol{R}_{\mathbf{N}}$.

\section{Appendix}

Throughout this section, we assume that $b_{1}=\cdots=b_{k}=1$, without loss of generality. Throughout, let $n_{*}=\sum_{i=1}^{k} n_{i}$. Let $\boldsymbol{y}_{j}=\left(\boldsymbol{x}_{1 j}-\boldsymbol{\mu}_{1}\right) / n_{1}$ for $j=1, \ldots, n_{1}$, and $\boldsymbol{y}_{j+\sum_{i^{\prime}=1}^{i-1} n_{i^{\prime}}}=\left(\boldsymbol{x}_{i j}-\boldsymbol{\mu}_{i}\right) / n_{i}$ for $j=1, \ldots, n_{i}(i \geq 2)$. Let $V_{n_{*} j}=\sum_{i=1}^{j-1} \boldsymbol{y}_{i}^{T} \boldsymbol{y}_{j}$ for $j=2, \ldots, n_{*}$. Note that $E_{\boldsymbol{\theta}}\left\{\left(2 \sum_{j=2}^{n_{*}} V_{n_{*} j}\right)^{2}\right\}=$ $2 \sum_{i=1}^{k} \operatorname{tr}\left(\boldsymbol{\Sigma}_{i}^{2}\right)\left(n_{i}-1\right) / n_{i}^{3}+4 \sum_{i<j} \operatorname{tr}\left(\boldsymbol{\Sigma}_{i} \boldsymbol{\Sigma}_{j}\right) /\left(n_{i} n_{j}\right)$ (= $K_{v}$, say).

Lemma 1 Assume (A-v) and (A-vi). Then, it holds as $p \rightarrow \infty$ and $n_{i} \rightarrow$ $\infty, i=1, \ldots, k$, that

$$
\frac{4 \sum_{j=2}^{n_{*}} V_{n_{*} j}^{2}}{K_{v}}=1+o_{p}(1) .
$$

Proof Let $\boldsymbol{\Gamma}_{i}=\left(\boldsymbol{\gamma}_{i 1}, \ldots, \boldsymbol{\gamma}_{i r_{i}}\right), i=1, \ldots, k$. Let $i_{*}$ be an integer such that $i \in\left[1+\sum_{j=1}^{i_{*}-1} n_{j}, \sum_{j=1}^{i_{*}} n_{j}\right]$, where $\sum_{j=1}^{0} n_{j}=0$. Note that $\operatorname{tr}\left(\boldsymbol{\Sigma}_{i} \boldsymbol{\Sigma}_{j} \boldsymbol{\Sigma}_{i^{\prime}} \boldsymbol{\Sigma}_{j}\right)=$ $\operatorname{tr}\left(\boldsymbol{\Sigma}_{j}^{1 / 2} \boldsymbol{\Sigma}_{i} \boldsymbol{\Sigma}_{j}^{1 / 2} \boldsymbol{\Sigma}_{j}^{1 / 2} \boldsymbol{\Sigma}_{i^{\prime}} \boldsymbol{\Sigma}_{j}^{1 / 2}\right) \leq\left\{\operatorname{tr}\left(\boldsymbol{\Sigma}_{i} \boldsymbol{\Sigma}_{j} \boldsymbol{\Sigma}_{i} \boldsymbol{\Sigma}_{j}\right) \operatorname{tr}\left(\boldsymbol{\Sigma}_{i^{\prime}} \boldsymbol{\Sigma}_{j} \boldsymbol{\Sigma}_{i^{\prime}} \boldsymbol{\Sigma}_{j}\right)\right\}^{1 / 2}$. Under (A-v), we have for $i \neq i^{\prime} \neq j$ that

$$
\begin{aligned}
& n_{i_{*}}^{2} n_{i_{*}^{\prime}}^{2} n_{j_{*}}^{4} E_{\boldsymbol{\theta}}\left\{\left(\boldsymbol{y}_{i}^{T} \boldsymbol{y}_{j}\right)^{2}\left(\boldsymbol{y}_{i^{\prime}}^{T} \boldsymbol{y}_{j}\right)^{2}\right\}=n_{j_{*}}^{4} E_{\boldsymbol{\theta}}\left(\boldsymbol{y}_{j}^{T} \boldsymbol{\Sigma}_{i_{*}} \boldsymbol{y}_{j} \boldsymbol{y}_{j}^{T} \boldsymbol{\Sigma}_{i_{*}^{\prime}} \boldsymbol{y}_{j}\right) \\
& =E_{\boldsymbol{\theta}}\left(\sum_{s, t, u, v}^{r_{j_{*}}} \gamma_{j_{*} s}^{T} \boldsymbol{\Sigma}_{i_{*}} \gamma_{j_{*} t} \gamma_{j_{*} u}^{T} \boldsymbol{\Sigma}_{i_{*}^{\prime}} \gamma_{j_{*} v} w_{j_{*} s j} w_{j_{*} t j} w_{j_{*} u j} w_{j_{*} v j}\right) \\
& =\operatorname{tr}\left(\boldsymbol{\Sigma}_{i_{*}} \boldsymbol{\Sigma}_{j_{*}}\right) \operatorname{tr}\left(\boldsymbol{\Sigma}_{i_{*}^{\prime}} \boldsymbol{\Sigma}_{j_{*}}\right)+2 \operatorname{tr}\left(\boldsymbol{\Sigma}_{i_{*}} \boldsymbol{\Sigma}_{j_{*}} \boldsymbol{\Sigma}_{i_{*}^{\prime}} \boldsymbol{\Sigma}_{j_{*}}\right) \\
& \quad+O\left\{\sum_{s=1}^{r_{j_{*}}} \gamma_{j_{*} s}^{T} \boldsymbol{\Sigma}_{i_{*}} \gamma_{j_{*} s} \gamma_{j_{*} s}^{T} \boldsymbol{\Sigma}_{i_{*}^{\prime}} \gamma_{j_{*} s}\right\} \\
& =\operatorname{tr}\left(\boldsymbol{\Sigma}_{i_{*}} \boldsymbol{\Sigma}_{j_{*}}\right) \operatorname{tr}\left(\boldsymbol{\Sigma}_{i_{*}^{\prime}} \boldsymbol{\Sigma}_{j_{*}}\right)+O\left[\left\{\operatorname{tr}\left(\boldsymbol{\Sigma}_{i_{*}} \boldsymbol{\Sigma}_{j_{*}} \boldsymbol{\Sigma}_{i_{*}} \boldsymbol{\Sigma}_{j_{*}}\right) \operatorname{tr}\left(\boldsymbol{\Sigma}_{i_{*}^{\prime}} \boldsymbol{\Sigma}_{j_{*}} \boldsymbol{\Sigma}_{i_{*}^{\prime}} \boldsymbol{\Sigma}_{j_{*}}\right)\right\}^{1 / 2}\right]
\end{aligned}
$$

from the fact that

$$
\begin{aligned}
& \sum_{s=1}^{r_{j_{*}}} \gamma_{j_{*} s}^{T} \boldsymbol{\Sigma}_{i_{*}} \gamma_{j_{*} s} \gamma_{j_{*} s}^{T} \boldsymbol{\Sigma}_{i_{*}^{\prime}} \gamma_{j_{*} s} \leq\left\{\sum_{s=1}^{r_{j_{*}}}\left(\gamma_{j_{*} s}^{T} \boldsymbol{\Sigma}_{i_{*}} \gamma_{j_{*} s}\right)^{2} \sum_{s^{\prime}=1}^{r_{j_{*}}}\left(\gamma_{j_{*} s^{\prime}}^{T} \boldsymbol{\Sigma}_{i_{*}^{\prime}} \gamma_{j_{*} s^{\prime}}\right)^{2}\right\}^{1 / 2} \\
& \leq\left\{\sum_{s, t}^{r_{j_{*}}}\left(\gamma_{j_{*} s}^{T} \boldsymbol{\Sigma}_{i_{*}} \gamma_{j_{*} t}\right)^{2} \sum_{s^{\prime}, t^{\prime}}^{r_{j_{*}}}\left(\boldsymbol{\gamma}_{j_{*} s^{\prime}}^{T} \boldsymbol{\Sigma}_{i_{*}^{\prime}} \gamma_{j_{*} t^{\prime}}\right)^{2}\right\}^{1 / 2} \\
& =\left\{\operatorname{tr}\left(\boldsymbol{\Sigma}_{i_{*}} \boldsymbol{\Sigma}_{j_{*}} \boldsymbol{\Sigma}_{i_{*}} \boldsymbol{\Sigma}_{j_{*}}\right) \operatorname{tr}\left(\boldsymbol{\Sigma}_{i_{*}^{\prime}} \boldsymbol{\Sigma}_{j_{*}} \boldsymbol{\Sigma}_{i_{*}^{\prime}} \boldsymbol{\Sigma}_{j_{*}}\right)\right\}^{1 / 2}
\end{aligned}
$$


On the other hand, from (15), under (A-v), we have for $i \neq j$ that

$$
\begin{aligned}
& E_{\boldsymbol{\theta}}\left\{\left(\boldsymbol{y}_{i}^{T} \boldsymbol{y}_{j}\right)^{4}\right\} n_{i_{*}}^{4} n_{j_{*}}^{4} \\
& =E_{\boldsymbol{\theta}}\left\{\left(\sum_{s, u}^{r_{i_{*}}} \sum_{t, v}^{r_{j_{*}}} \gamma_{i_{*} s}^{T} \gamma_{j_{*} t} w_{i_{*} s i} w_{j_{*} t j} \gamma_{i_{*} u}^{T} \gamma_{j_{*} v} w_{i_{*} u i} w_{j_{*} v j}\right)^{2}\right\} \\
& =\operatorname{tr}\left(\boldsymbol{\Sigma}_{i_{*}} \boldsymbol{\Sigma}_{j_{*}}\right)^{2}+E_{\boldsymbol{\theta}}\left\{\left(\sum_{s \neq u}^{r_{i_{*}}} \sum_{t \neq v}^{r_{j_{*}}} \gamma_{i_{*} s}^{T} \gamma_{j_{*} t} w_{i_{*} s i} w_{j_{*} t j} \gamma_{i_{*} u}^{T} \gamma_{j_{*} v} w_{i_{*} u i} w_{j_{*} v j}\right)^{2}\right\} \\
& \quad+O\left\{\operatorname{tr}\left(\boldsymbol{\Sigma}_{i_{*}} \boldsymbol{\Sigma}_{j_{*}} \boldsymbol{\Sigma}_{i_{*}} \boldsymbol{\Sigma}_{j_{*}}\right)\right\}+O\left(\sum_{s=1}^{r_{j_{*}}} \gamma_{j_{*} s}^{T} \boldsymbol{\Sigma}_{i_{*}} \gamma_{j_{*} s} \gamma_{j_{*} s}^{T} \boldsymbol{\Sigma}_{i_{*}} \boldsymbol{\gamma}_{j_{*} s}\right) \\
& \quad+O\left(\sum_{s=1}^{r_{i_{*}}} \gamma_{i_{*} s}^{T} \boldsymbol{\Sigma}_{j_{*}} \gamma_{i_{*} s} \boldsymbol{\gamma}_{i_{*} s}^{T} \boldsymbol{\Sigma}_{j_{*}} \gamma_{i_{*} s}\right) \\
& =3 \operatorname{tr}\left(\boldsymbol{\Sigma}_{i_{*}} \boldsymbol{\Sigma}_{j_{*}}\right)^{2}+O\left\{\operatorname{tr}\left(\boldsymbol{\Sigma}_{i_{*}} \boldsymbol{\Sigma}_{j_{*}} \boldsymbol{\Sigma}_{i_{*}} \boldsymbol{\Sigma}_{j_{*}}\right)\right\}=O\left\{\operatorname{tr}\left(\boldsymbol{\Sigma}_{i_{*}} \boldsymbol{\Sigma}_{j_{*}}\right)^{2}\right\}
\end{aligned}
$$

from the fact that $\operatorname{tr}\left(\boldsymbol{\Sigma}_{i} \boldsymbol{\Sigma}_{j} \boldsymbol{\Sigma}_{i} \boldsymbol{\Sigma}_{j}\right)=\operatorname{tr}\left\{\left(\boldsymbol{\Sigma}_{i}^{1 / 2} \boldsymbol{\Sigma}_{j} \boldsymbol{\Sigma}_{i}^{1 / 2}\right)^{2}\right\} \leq \operatorname{tr}\left(\boldsymbol{\Sigma}_{i} \boldsymbol{\Sigma}_{j}\right)^{2}$. Let $\boldsymbol{B}_{j}=\boldsymbol{\Sigma}_{j^{\prime}} / n_{j^{\prime}}^{2}$ for $j \in\left[1+\sum_{i=1}^{j^{\prime}-1} n_{i}, \sum_{i=1}^{j^{\prime}} n_{i}\right]$, where $\sum_{i=1}^{0} n_{i}=0$. Note that $E_{\boldsymbol{\theta}}\left(V_{n_{*} j}^{2}\right)=\sum_{i=1}^{j-1} \operatorname{tr}\left(\boldsymbol{B}_{i} \boldsymbol{B}_{j}\right)$ for $j=2, \ldots, n_{*}$, and $4 \sum_{j=2}^{n_{*}} \sum_{i=1}^{j-1} \operatorname{tr}\left(\boldsymbol{B}_{i} \boldsymbol{B}_{j}\right)=$ $K_{v}$. From (14)-(16), we have for $2 \leq j<j^{\prime}$ that

$$
\begin{aligned}
& E_{\boldsymbol{\theta}}\left[\left\{V_{n_{*} j}^{2}-E_{\boldsymbol{\theta}}\left(V_{n_{*} j}^{2}\right)\right\}^{2}\right] \leq E_{\boldsymbol{\theta}}\left(V_{n_{*} j}^{4}\right)=O\left\{\sum_{i, i^{\prime}}^{j-1} \operatorname{tr}\left(\boldsymbol{B}_{i} \boldsymbol{B}_{j}\right) \operatorname{tr}\left(\boldsymbol{B}_{i^{\prime}} \boldsymbol{B}_{j}\right)\right\} ; \\
& E_{\boldsymbol{\theta}}\left[\left\{V_{n_{*} j}^{2}-E_{\boldsymbol{\theta}}\left(V_{n_{*} j}^{2}\right)\right\}\left\{V_{n_{*} j^{\prime}}^{2}-E_{\boldsymbol{\theta}}\left(V_{n_{*} j^{\prime}}^{2}\right)\right\}\right] \\
& =O\left[\sum_{i, i^{\prime}}^{j-1}\left\{\operatorname{tr}\left(\boldsymbol{B}_{i} \boldsymbol{B}_{j} \boldsymbol{B}_{i} \boldsymbol{B}_{j}\right) \operatorname{tr}\left(\boldsymbol{B}_{i^{\prime}} \boldsymbol{B}_{j^{\prime}} \boldsymbol{B}_{i^{\prime}} \boldsymbol{B}_{j^{\prime}}\right)\right\}^{1 / 2}\right] \\
& +O\left[\sum_{i=1}^{j-1} \operatorname{tr}\left(\boldsymbol{B}_{i} \boldsymbol{B}_{j}\right)\left\{\operatorname{tr}\left(\boldsymbol{B}_{i} \boldsymbol{B}_{j^{\prime}}\right)+\operatorname{tr}\left(\boldsymbol{B}_{j} \boldsymbol{B}_{j^{\prime}}\right)\right\}\right]
\end{aligned}
$$

from the fact that

$$
\begin{aligned}
E_{\boldsymbol{\theta}}\left\{\left(\boldsymbol{y}_{i}^{T} \boldsymbol{y}_{j}\right)^{2}\left(\boldsymbol{y}_{i}^{T} \boldsymbol{y}_{j^{\prime}}\right)\left(\boldsymbol{y}_{j}^{T} \boldsymbol{y}_{j^{\prime}}\right)\right\} & \leq\left[E_{\boldsymbol{\theta}}\left\{\left(\boldsymbol{y}_{i}^{T} \boldsymbol{y}_{j}\right)^{4}\right\} E_{\boldsymbol{\theta}}\left\{\left(\boldsymbol{y}_{i}^{T} \boldsymbol{y}_{j^{\prime}}\right)^{2}\left(\boldsymbol{y}_{j}^{T} \boldsymbol{y}_{j^{\prime}}\right)^{2}\right\}\right]^{1 / 2} \\
& =O\left[\operatorname{tr}\left(\boldsymbol{B}_{i} \boldsymbol{B}_{j}\right)\left\{\operatorname{tr}\left(\boldsymbol{B}_{i} \boldsymbol{B}_{j^{\prime}}\right) \operatorname{tr}\left(\boldsymbol{B}_{j} \boldsymbol{B}_{j^{\prime}}\right)\right\}^{1 / 2}\right] \\
& =O\left[\operatorname{tr}\left(\boldsymbol{B}_{i} \boldsymbol{B}_{j}\right)\left\{\operatorname{tr}\left(\boldsymbol{B}_{i} \boldsymbol{B}_{j^{\prime}}\right)+\operatorname{tr}\left(\boldsymbol{B}_{j} \boldsymbol{B}_{j^{\prime}}\right)\right\}\right]
\end{aligned}
$$

for $i<j<j^{\prime}$. Then, from (17), we can obtain as $n_{i} \rightarrow \infty, i=1, \ldots, k$, that

$$
\sum_{j=2}^{n_{*}} E_{\boldsymbol{\theta}}\left[\left\{V_{n_{*} j}^{2}-E_{\boldsymbol{\theta}}\left(V_{n_{*} j}^{2}\right)\right\}^{2}\right] \leq \sum_{j=2}^{n_{*}} E_{\boldsymbol{\theta}}\left(V_{n_{*} j}^{4}\right)=O\left(\frac{K_{v}^{2}}{\min _{i=1, \ldots, k} n_{i}}\right)=o\left(K_{v}^{2}\right) .
$$


On the other hand, under (A-vi), we have for all $i, i^{\prime}, j, j^{\prime}$ that

$$
\begin{aligned}
\frac{\left\{\operatorname{tr}\left(\boldsymbol{\Sigma}_{i} \boldsymbol{\Sigma}_{j} \boldsymbol{\Sigma}_{i} \boldsymbol{\Sigma}_{j}\right) \operatorname{tr}\left(\boldsymbol{\Sigma}_{i^{\prime}} \boldsymbol{\Sigma}_{j^{\prime}} \boldsymbol{\Sigma}_{i^{\prime}} \boldsymbol{\Sigma}_{j^{\prime}}\right)\right\}^{1 / 2}}{\left\{\operatorname{tr}\left(\boldsymbol{\Sigma}_{i}^{2}\right) \operatorname{tr}\left(\boldsymbol{\Sigma}_{j}^{2}\right) \operatorname{tr}\left(\boldsymbol{\Sigma}_{i^{\prime}}^{2}\right) \operatorname{tr}\left(\boldsymbol{\Sigma}_{j^{\prime}}^{2}\right)\right\}^{1 / 2}} & \leq \frac{\left\{\operatorname{tr}\left(\boldsymbol{\Sigma}_{i}^{4}\right) \operatorname{tr}\left(\boldsymbol{\Sigma}_{j}^{4}\right) \operatorname{tr}\left(\boldsymbol{\Sigma}_{i^{\prime}}^{4}\right) \operatorname{tr}\left(\boldsymbol{\Sigma}_{j^{\prime}}^{4}\right)\right\}^{1 / 4}}{\left\{\operatorname{tr}\left(\boldsymbol{\Sigma}_{i}^{2}\right) \operatorname{tr}\left(\boldsymbol{\Sigma}_{j}^{2}\right) \operatorname{tr}\left(\boldsymbol{\Sigma}_{i^{\prime}}^{2}\right) \operatorname{tr}\left(\boldsymbol{\Sigma}_{j^{\prime}}^{2}\right)\right\}^{1 / 2}} \\
& \leq \frac{\left\{\lambda_{i 1} \lambda_{j 1} \lambda_{i^{\prime} 1} \lambda_{j^{\prime} 1}\right\}^{1 / 2}}{\left\{\operatorname{tr}\left(\boldsymbol{\Sigma}_{i}^{2}\right) \operatorname{tr}\left(\boldsymbol{\Sigma}_{j}^{2}\right) \operatorname{tr}\left(\boldsymbol{\Sigma}_{i^{\prime}}^{2}\right) \operatorname{tr}\left(\boldsymbol{\Sigma}_{j^{\prime}}^{2}\right)\right\}^{1 / 4}} \\
& \rightarrow 0
\end{aligned}
$$

from the facts that $\operatorname{tr}\left(\boldsymbol{\Sigma}_{i} \boldsymbol{\Sigma}_{j} \boldsymbol{\Sigma}_{i} \boldsymbol{\Sigma}_{j}\right) \leq \operatorname{tr}\left(\boldsymbol{\Sigma}_{i}^{2} \boldsymbol{\Sigma}_{j}^{2}\right) \leq \operatorname{tr}\left(\boldsymbol{\Sigma}_{i}^{4}\right)^{1 / 2} \operatorname{tr}\left(\boldsymbol{\Sigma}_{j}^{4}\right)^{1 / 2}$ and $\operatorname{tr}\left(\boldsymbol{\Sigma}_{i}^{4}\right)^{1 / 2} \leq \lambda_{i 1} \operatorname{tr}\left(\boldsymbol{\Sigma}_{i}^{2}\right)^{1 / 2}$. Then, it holds under (A-vi) that

$$
\begin{aligned}
& \frac{\left\{\operatorname{tr}\left(\boldsymbol{\Sigma}_{i} \boldsymbol{\Sigma}_{j} \boldsymbol{\Sigma}_{i} \boldsymbol{\Sigma}_{j}\right) \operatorname{tr}\left(\boldsymbol{\Sigma}_{i^{\prime}} \boldsymbol{\Sigma}_{j^{\prime}} \boldsymbol{\Sigma}_{i^{\prime}} \boldsymbol{\Sigma}_{j^{\prime}}\right)\right\}^{1 / 2} /\left(n_{i} n_{j} n_{i^{\prime}} n_{j^{\prime}}\right)}{K_{v}^{2}} \\
& =O\left[\frac{\left\{\operatorname{tr}\left(\boldsymbol{\Sigma}_{i} \boldsymbol{\Sigma}_{j} \boldsymbol{\Sigma}_{i} \boldsymbol{\Sigma}_{j}\right) \operatorname{tr}\left(\boldsymbol{\Sigma}_{i^{\prime}} \boldsymbol{\Sigma}_{j^{\prime}} \boldsymbol{\Sigma}_{i^{\prime}} \boldsymbol{\Sigma}_{j^{\prime}}\right)\right\}^{1 / 2} /\left(n_{i} n_{j} n_{i^{\prime}} n_{j^{\prime}}\right)}{\left.\left\{\operatorname{tr}\left(\boldsymbol{\Sigma}_{i}^{2}\right) / n_{i}^{2}+\operatorname{tr}\left(\boldsymbol{\Sigma}_{j}^{2}\right) / n_{j}^{2}\right\} \operatorname{tr}\left(\boldsymbol{\Sigma}_{i^{\prime}}^{2}\right) / n_{i^{\prime}}^{2}+\operatorname{tr}\left(\boldsymbol{\Sigma}_{j^{\prime}}^{2}\right) / n_{j^{\prime}}^{2}\right\}}\right] \\
& =O\left[\frac{\left\{\operatorname{tr}\left(\boldsymbol{\Sigma}_{i} \boldsymbol{\Sigma}_{j} \boldsymbol{\Sigma}_{i} \boldsymbol{\Sigma}_{j}\right) \operatorname{tr}\left(\boldsymbol{\Sigma}_{i^{\prime}} \boldsymbol{\Sigma}_{j^{\prime}} \boldsymbol{\Sigma}_{i^{\prime}} \boldsymbol{\Sigma}_{j^{\prime}}\right)\right\}^{1 / 2}}{\left\{\operatorname{tr}\left(\boldsymbol{\Sigma}_{i}^{2}\right) \operatorname{tr}\left(\boldsymbol{\Sigma}_{j}^{2}\right) \operatorname{tr}\left(\boldsymbol{\Sigma}_{i^{\prime}}^{2}\right) \operatorname{tr}\left(\boldsymbol{\Sigma}_{j^{\prime}}^{2}\right)\right\}^{1 / 2}}\right] \rightarrow 0 .
\end{aligned}
$$

Hence, from (18), under (A-vi), we can claim that

$$
\sum_{2 \leq j<j^{\prime}}^{n_{*}} E_{\boldsymbol{\theta}}\left[\left\{V_{n_{*} j}^{2}-E_{\boldsymbol{\theta}}\left(V_{n_{*} j}^{2}\right)\right\}\left\{V_{n_{*} j^{\prime}}^{2}-E_{\boldsymbol{\theta}}\left(V_{n_{*} j^{\prime}}^{2}\right)\right\}\right]=o\left(K_{v}^{2}\right) \text {. }
$$

Thus by combining (19) and (20), we have as $p \rightarrow \infty$ and $n_{i} \rightarrow \infty, i=1, \ldots, k$, that

$$
\operatorname{Var}_{\boldsymbol{\theta}}\left(\sum_{j=2}^{n_{*}} V_{n_{*} j}^{2}\right)=o\left(K_{v}^{2}\right)
$$

under $(\mathrm{A}-\mathrm{v})$ and $(\mathrm{A}-\mathrm{vi})$. Thus by using Chebyshev's inequality, from the fact that $4 \sum_{j=2}^{n_{*}} E_{\boldsymbol{\theta}}\left(V_{n_{*} j}^{2}\right)=K_{v}$, it holds that $4 \sum_{j=2}^{n_{*}} V_{n_{*} j}^{2} / K_{v}=1+o_{p}(1)$. Thus it concludes the result.

Lemma 2 Let $I(\cdot)$ be the indicator function. For Lindeberg's condition, under $(A-v)$, it holds as $p \rightarrow \infty$ and $n_{i} \rightarrow \infty, i=1, \ldots, k$, that

$$
\sum_{j=2}^{n_{*}} E_{\boldsymbol{\theta}}\left\{\frac{V_{n_{*} j}^{2}}{K_{v}} I\left(\frac{V_{n_{*} j}^{2}}{K_{v}}>\tau\right)\right\} \rightarrow 0
$$

for any $\tau>0$.

Proof By using Chebyshev's inequality and Schwarz's inequality, we have that

$$
\begin{aligned}
\sum_{j=2}^{n_{*}} E_{\boldsymbol{\theta}}\left\{\frac{V_{n_{*} j}^{2}}{K_{v}} I\left(\frac{V_{n_{*} j}^{2}}{K_{v}}>\tau\right)\right\} & \leq \sum_{j=2}^{n_{*}}\left[E_{\boldsymbol{\theta}}\left(\frac{V_{n_{*} j}^{4}}{K_{v}^{2}}\right) E_{\boldsymbol{\theta}}\left\{I\left(\frac{V_{n_{*} j}^{2}}{K_{v}}>\tau\right)\right\}\right]^{1 / 2} \\
& \leq \tau^{-1} K_{v}^{-2} \sum_{j=2}^{n_{*}} E_{\boldsymbol{\theta}}\left(V_{n_{*} j}^{4}\right)
\end{aligned}
$$

Then, by combining (21) with (19), we can conclude the result. 
Lemma 3 Assume $(A-v)$. Then, it holds as $p \rightarrow \infty$ and $n_{i} \rightarrow \infty, i=1, \ldots, k$, that

$$
\frac{\widehat{K}}{K}=1+o_{p}(1)
$$

Proof From (14) and (16), we have for $i \neq j$ that

$$
\begin{aligned}
& E_{\boldsymbol{\theta}}\left[\left\{\left(\left(\boldsymbol{x}_{i s}-\boldsymbol{\mu}_{i}\right)^{T}\left(\boldsymbol{x}_{j t}-\boldsymbol{\mu}_{j}\right)\right)^{2}-\operatorname{tr}\left(\boldsymbol{\Sigma}_{i} \boldsymbol{\Sigma}_{j}\right)\right\}^{2}\right]=O\left\{\operatorname{tr}\left(\boldsymbol{\Sigma}_{i} \boldsymbol{\Sigma}_{j}\right)^{2}\right\} \\
& E_{\boldsymbol{\theta}}\left[\left\{\left(\boldsymbol{x}_{i s}-\boldsymbol{\mu}_{i}\right)^{T}\left(\boldsymbol{x}_{j t}-\boldsymbol{\mu}_{j}\right)\right\}^{2}\left\{\left(\boldsymbol{x}_{i s^{\prime}}-\boldsymbol{\mu}_{i}\right)^{T}\left(\boldsymbol{x}_{j t}-\boldsymbol{\mu}_{j}\right)\right\}^{2}-\operatorname{tr}\left(\boldsymbol{\Sigma}_{i} \boldsymbol{\Sigma}_{j}\right)^{2}\right] \\
& =O\left\{\operatorname{tr}\left(\boldsymbol{\Sigma}_{i} \boldsymbol{\Sigma}_{j} \boldsymbol{\Sigma}_{i} \boldsymbol{\Sigma}_{j}\right)\right\} \quad\left(s \neq s^{\prime}\right)
\end{aligned}
$$

under $(\mathrm{A}-\mathrm{v})$. We write that

$\operatorname{tr}\left(\boldsymbol{S}_{i n_{i}} \boldsymbol{S}_{j n_{j}}\right)=\sum_{s=1}^{n_{i}} \sum_{t=1}^{n_{j}} \frac{\left[\left\{\boldsymbol{x}_{i s}-\boldsymbol{\mu}_{i}-\left(\overline{\boldsymbol{x}}_{i n_{i}}-\boldsymbol{\mu}_{i}\right)\right\}^{T}\left\{\boldsymbol{x}_{j t}-\boldsymbol{\mu}_{j}-\left(\overline{\boldsymbol{x}}_{j n_{j}}-\boldsymbol{\mu}_{j}\right)\right\}\right]^{2}}{\left(n_{i}-1\right)\left(n_{j}-1\right)}$.

Then, from (22), it holds as $p \rightarrow \infty$ and $n_{i} \rightarrow \infty, i=1, \ldots, k$, that

$$
\operatorname{Var}_{\boldsymbol{\theta}}\left\{\operatorname{tr}\left(\boldsymbol{S}_{i n_{i}} \boldsymbol{S}_{j n_{j}}\right)\right\}=o\left\{\operatorname{tr}\left(\boldsymbol{\Sigma}_{i} \boldsymbol{\Sigma}_{j}\right)^{2}\right\}
$$

for $i<j$. By combining (5) with (23), we can claim under (A-v) that $\widehat{K}=$ $K\left\{1+o_{p}(1)\right\}$ as $p \rightarrow \infty$ and $n_{i} \rightarrow \infty, i=1, \ldots, k$. Thus it concludes the result.

Proofs of Theorem 4 and Corollary 4 Define $S_{n_{*} t}=\sum_{j=2}^{t} V_{n_{*} j}$ for $t=2, \ldots, n_{*}$. Let $\mathcal{F}_{n_{*} i}=\sigma\left(\boldsymbol{y}_{1}, \boldsymbol{y}_{2}, \ldots, \boldsymbol{y}_{i}\right)$ be the $\sigma$ algebra by $\left\{\boldsymbol{y}_{1}, \boldsymbol{y}_{2}, \ldots, \boldsymbol{y}_{i}\right\}$ for $i \geq 2$. Note that $S_{n_{*} t}$ is of zero mean and square integrable. Note that $E_{\boldsymbol{\theta}}\left(S_{n_{*} t} \mid \mathcal{F}_{i}\right)=$ $\sum_{j=2}^{i} V_{n_{*} j}=S_{n_{*} i}$ for $t>i$. Thus $\left\{S_{n_{*} i}, \mathcal{F}_{n_{*} i}\right\}_{i=2}^{n_{*}}$ is a sequence of zero mean and a square integrable martingale. We consider applying the martingale central limit theorem given by McLeish (1974). Refer to Section 2.6 in Ghosh et al. (1997) for the details of the martingale central limit theorem. We have as $n_{i} \rightarrow \infty, i=1, \ldots, k$, that

$$
\begin{aligned}
& \left\|\boldsymbol{T}_{\mathbf{n}}-\boldsymbol{\mu}\right\|^{2}-\widehat{\Sigma}_{\mathbf{n}} \\
& =2 \sum_{i=1}^{k} \sum_{s<t} \frac{\left(\boldsymbol{x}_{i s}-\boldsymbol{\mu}\right)^{T}\left(\boldsymbol{x}_{i t}-\boldsymbol{\mu}\right)}{n_{i}\left(n_{i}-1\right)}+2 \sum_{i<j} \sum_{s=1}^{n_{i}} \sum_{t=1}^{n_{j}} \frac{\left(\boldsymbol{x}_{i s}-\boldsymbol{\mu}\right)^{T}\left(\boldsymbol{x}_{j t}-\boldsymbol{\mu}\right)}{n_{i} n_{j}} \\
& =2 \sum_{j=2}^{n_{*}} V_{n_{*} j}+2 \sum_{i=1}^{k} \sum_{s<t} \frac{\left(\boldsymbol{x}_{i s}-\boldsymbol{\mu}\right)^{T}\left(\boldsymbol{x}_{i t}-\boldsymbol{\mu}\right)}{n_{i}^{2}\left(n_{i}-1\right)}=2 \sum_{j=2}^{n_{*}} V_{n_{*} j}+o_{p}\left(K^{1 / 2}\right) .
\end{aligned}
$$

Note that $K / K_{v} \rightarrow 1$ as $n_{i} \rightarrow \infty, i=1, \ldots, k$. Then, by using the martingale central limit theorem, we obtain that

$$
\frac{\left\|\boldsymbol{T}_{\mathbf{n}}-\boldsymbol{\mu}\right\|^{2}-\widehat{\Sigma}_{\mathbf{n}}}{K}=\frac{2 \sum_{j=2}^{n_{*}} V_{n_{*} j}}{K_{v}}+o_{p}(1) \Rightarrow N(0,1)
$$

by combining Lemmas 1 and 2 with (24). Thus it concludes the result of Theorem 4. On the other hand, by using Lemma 3, we obtain from (25) that 
$\left(\left\|\boldsymbol{T}_{\mathbf{n}}-\boldsymbol{\mu}\right\|^{2}-\widehat{\Sigma}_{\mathbf{n}}\right) / \widehat{K} \Rightarrow N(0,1)$. It concludes the result of Corollary 4.

Proofs of Theorem 5 and Corollary 5 Note that

$$
\begin{aligned}
\widehat{T}_{\mathbf{n}}-\|\boldsymbol{\mu}\|^{2} & =\left\|\boldsymbol{T}_{\mathbf{n}}-\boldsymbol{\mu}\right\|^{2}-\widehat{\Sigma}_{\mathbf{n}}+2\left(\boldsymbol{T}_{\mathbf{n}}-\boldsymbol{\mu}\right)^{T} \boldsymbol{\mu} \\
& =\left\|\boldsymbol{T}_{\mathbf{n}}-\boldsymbol{\mu}\right\|^{2}-\widehat{\Sigma}_{\mathbf{n}}+o_{p}\left(K^{1 / 2}\right)
\end{aligned}
$$

and $K_{*} / K \rightarrow 1$ under (A-vii). Thus in a way similar to the proofs of Theorem 4 and Corollary 4 , we can conclude the results.

Proof of Corollary 2 Note that $\sum_{i=1}^{k} \lambda_{i 1} /\left(n_{i} K^{1 / 2}\right)=O\left\{\sum_{i=1}^{k} \lambda_{i 1} / \operatorname{tr}\left(\boldsymbol{\Sigma}_{i}^{2}\right)^{1 / 2}\right\}$ $=o(1)$ under $(\mathrm{A}-\mathrm{vi})$. Then, it holds under $(\mathrm{A}-\mathrm{vi})$ that

$$
\sum_{i=1}^{k} \frac{\boldsymbol{\mu}^{T} \boldsymbol{\Sigma}_{i} \boldsymbol{\mu}}{n_{i} K} \leq\|\boldsymbol{\mu}\|^{2} \sum_{i=1}^{k} \frac{\lambda_{i 1}}{n_{i} K}=\frac{\|\boldsymbol{\mu}\|^{2}}{K^{1 / 2}} \sum_{i=1}^{k} \frac{\lambda_{i 1}}{n_{i} K^{1 / 2}}=\frac{\|\boldsymbol{\mu}\|^{2}}{K^{1 / 2}} \times o(1)
$$

so that we obtain that $K /\|\boldsymbol{\mu}\|^{4}=o(1)$ under (A-vi) and (A-viii). Then, it holds that $\sum_{i=1}^{k} \boldsymbol{\mu}^{T} \boldsymbol{\Sigma}_{i} \boldsymbol{\mu} /\left(n_{i}\|\boldsymbol{\mu}\|^{4}\right) \leq \sum_{i=1}^{k} \lambda_{i 1} /\left(n_{i}\|\boldsymbol{\mu}\|^{2}\right) \leq \sum_{i=1}^{k} \operatorname{tr}\left(\boldsymbol{\Sigma}_{i}^{2}\right)^{1 / 2}$ $/\left(n_{i}\|\boldsymbol{\mu}\|^{2}\right)=O\left(K^{1 / 2} /\|\boldsymbol{\mu}\|^{2}\right)=o(1)$. Thus we have that $\operatorname{Var}_{\boldsymbol{\theta}}\left(\widehat{T}_{\mathbf{n}}\right) /\|\boldsymbol{\mu}\|^{4}=$ $K_{*} /\|\boldsymbol{\mu}\|^{4}=o(1)$ under (A-vi) and (A-viii). It concludes the result.

Proof of Corollary 3 By using Schwarz's inequality, it holds under (A-v') that $E_{\boldsymbol{\theta}}\left[\left\{\left(\boldsymbol{x}_{i l}-\boldsymbol{\mu}_{i}\right)^{T} \boldsymbol{\Sigma}_{j}\left(\boldsymbol{x}_{i l}-\boldsymbol{\mu}_{i}\right)-\operatorname{tr}\left(\boldsymbol{\Sigma}_{i} \boldsymbol{\Sigma}_{j}\right)\right\}\left\{\left(\boldsymbol{x}_{i l}-\boldsymbol{\mu}_{i}\right)^{T} \boldsymbol{\Sigma}_{j^{\prime}}\left(\boldsymbol{x}_{i l}-\boldsymbol{\mu}_{i}\right)-\right.\right.$ $\left.\left.\operatorname{tr}\left(\boldsymbol{\Sigma}_{i} \boldsymbol{\Sigma}_{j^{\prime}}\right)\right\}\right] \leq\left[\operatorname{Var}_{\boldsymbol{\theta}}\left\{\left(\boldsymbol{x}_{i l}-\boldsymbol{\mu}_{i}\right)^{T} \boldsymbol{\Sigma}_{j}\left(\boldsymbol{x}_{i l}-\boldsymbol{\mu}_{i}\right)\right\} \operatorname{Var}_{\boldsymbol{\theta}}\left\{\left(\boldsymbol{x}_{i l}-\boldsymbol{\mu}_{i}\right)^{T} \boldsymbol{\Sigma}_{j^{\prime}}\left(\boldsymbol{x}_{i l}-\right.\right.\right.$ $\left.\left.\left.\boldsymbol{\mu}_{i}\right)\right\}\right]^{1 / 2}=O\left[\left\{\operatorname{tr}\left(\boldsymbol{\Sigma}_{i} \boldsymbol{\Sigma}_{j} \boldsymbol{\Sigma}_{i} \boldsymbol{\Sigma}_{j}\right) \operatorname{tr}\left(\boldsymbol{\Sigma}_{i} \boldsymbol{\Sigma}_{j^{\prime}} \boldsymbol{\Sigma}_{i} \boldsymbol{\Sigma}_{j^{\prime}}\right)\right\}^{1 / 2}\right]$ for $i, j, j^{\prime}=1, \ldots, k$. Then, we have under (A-v') that

$$
\begin{aligned}
& n_{j_{*}}^{4} E_{\boldsymbol{\theta}}\left\{\boldsymbol{y}_{j}^{T} \boldsymbol{\Sigma}_{i_{*}} \boldsymbol{y}_{j} \boldsymbol{y}_{j}^{T} \boldsymbol{\Sigma}_{i_{*}^{\prime}} \boldsymbol{y}_{j}\right\}-\operatorname{tr}\left(\boldsymbol{\Sigma}_{i_{*}} \boldsymbol{\Sigma}_{j_{*}}\right) \operatorname{tr}\left(\boldsymbol{\Sigma}_{i_{*}^{\prime}} \boldsymbol{\Sigma}_{j_{*}}\right) \\
& =E_{\boldsymbol{\theta}}\left[\left\{n_{j_{*}}^{2} \boldsymbol{y}_{j}^{T} \boldsymbol{\Sigma}_{i_{*}} \boldsymbol{y}_{j}-\operatorname{tr}\left(\boldsymbol{\Sigma}_{i_{*}} \boldsymbol{\Sigma}_{j_{*}}\right)\right\}\left\{n_{j_{*}}^{2} \boldsymbol{y}_{j}^{T} \boldsymbol{\Sigma}_{i_{*}^{\prime}} \boldsymbol{y}_{j}-\operatorname{tr}\left(\boldsymbol{\Sigma}_{i_{*}^{\prime}} \boldsymbol{\Sigma}_{j_{*}}\right)\right\}\right] \\
& =O\left[\left\{\operatorname{tr}\left(\boldsymbol{\Sigma}_{i_{*}} \boldsymbol{\Sigma}_{j_{*}} \boldsymbol{\Sigma}_{i_{*}} \boldsymbol{\Sigma}_{j_{*}}\right) \operatorname{tr}\left(\boldsymbol{\Sigma}_{i_{*}^{\prime}} \boldsymbol{\Sigma}_{j_{*}} \boldsymbol{\Sigma}_{i_{*}^{\prime}} \boldsymbol{\Sigma}_{j_{*}}\right)\right\}^{1 / 2}\right]
\end{aligned}
$$

so that (14) holds. On the other hand, under (A-v'), it holds for $i \neq j$ that $n_{i_{*}}^{4} n_{j_{*}}^{4} E_{\boldsymbol{\theta}}\left\{\left(\boldsymbol{y}_{i}^{T} \boldsymbol{y}_{j}\right)^{4}\right\}=\operatorname{Var}_{\boldsymbol{\theta}}\left\{n_{i_{*}}^{2} n_{j_{*}}^{2}\left(\boldsymbol{y}_{i}^{T} \boldsymbol{y}_{j}\right)^{2}\right\}+\operatorname{tr}\left(\boldsymbol{\Sigma}_{i_{*}} \boldsymbol{\Sigma}_{j_{*}}\right)^{2}=O\left\{\operatorname{tr}\left(\boldsymbol{\Sigma}_{i_{*}} \boldsymbol{\Sigma}_{j_{*}}\right)^{2}\right\}$, so that (16) holds. Thus we claim Lemmas 1 and 2 after replacing (A-v) with (A-v'). We can conclude the result in a way similar to the proofs of Theorems 4 and 5.

Proof of Theorem 7 We have from (10) that $K^{1 / 2} \leq \delta / z_{\alpha / 2}$. Then, from 
Theorem 4 , we claim as $p \rightarrow \infty$ that

$$
\begin{aligned}
P_{\boldsymbol{\theta}}\left(\boldsymbol{\mu} \in \boldsymbol{R}_{\mathbf{n}}\right) & =P_{\boldsymbol{\theta}}\left(\max \left\{-\delta+\widehat{\Sigma}_{\mathbf{n}}, 0\right\} \leq\left\|\boldsymbol{T}_{\mathbf{n}}-\boldsymbol{\mu}\right\|^{2} \leq \delta+\widehat{\Sigma}_{\mathbf{n}}\right) \\
& =P_{\boldsymbol{\theta}}\left(\frac{-\delta}{K^{1 / 2}} \leq \frac{\left\|\boldsymbol{T}_{\mathbf{n}}-\boldsymbol{\mu}\right\|^{2}-\widehat{\Sigma}_{\mathbf{n}}}{K^{1 / 2}} \leq \frac{\delta}{K^{1 / 2}}\right) \\
& =P_{\boldsymbol{\theta}}\left(\left|\frac{\left\|\boldsymbol{T}_{\mathbf{n}}-\boldsymbol{\mu}\right\|^{2}-\widehat{\Sigma}_{\mathbf{n}}}{K^{1 / 2}}\right| \leq \frac{\delta}{K^{1 / 2}}\right) \\
& \geq P_{\boldsymbol{\theta}}\left(\left|\frac{\left\|\boldsymbol{T}_{\mathbf{n}}-\boldsymbol{\mu}\right\|^{2}-\widehat{\Sigma}_{\mathbf{n}}}{K^{1 / 2}}\right| \leq z_{\alpha / 2}\right) \\
& =P_{\boldsymbol{\theta}}\left(|N(0,1)|<z_{\alpha / 2}\right)+o(1)=1-\alpha+o(1)
\end{aligned}
$$

under $(\mathrm{A}-\mathrm{v})$ and $(\mathrm{A}-\mathrm{vi})$. Thus the proof is completed.

Proof of Theorem 8 From (5) and (12), it holds as $p \rightarrow \infty$ that $\left|N_{i}-C_{i}\right|=$ $o_{p}\left(C_{i}^{1 / 2}\right)$ under $(\mathrm{A}-\mathrm{v})$ and (A-vi). Then, we write that $\left|N_{i}-C_{i}\right|=O_{p}\left(\omega C_{i}^{1 / 2}\right)$, where $\omega(>0)$ is a variable such that $\omega \rightarrow 0$ as $p \rightarrow \infty$. Let $C_{i L}=\left\lfloor C_{i}-\right.$ $\left.\left(\omega C_{i}\right)^{1 / 2}\right\rfloor, i=1, \ldots, k$. We claim as $p \rightarrow \infty$ that $\max \left\{m_{i}, C_{i L}\right\} \leq N_{i}<$ $C_{i}+\left(\omega C_{i}\right)^{1 / 2}$ w.p.1. Then, in a way similar to the proofs of Theorems 2.4 and 2.5 in Aoshima and Yata (2011a), we claim that

$$
\left\|\boldsymbol{T}_{\mathbf{N}}-\boldsymbol{\mu}\right\|^{2}-\widehat{\Sigma}_{\mathbf{N}}=\left\|\boldsymbol{T}_{\mathbf{C}_{L}}-\boldsymbol{\mu}\right\|^{2}-\widehat{\Sigma}_{\mathbf{C}_{L}}+o_{p}(\delta),
$$

where $C_{L}=\left(C_{1 L}, \ldots, C_{k L}\right)$. From the fact that $C_{i L} / C_{i} \rightarrow 1$ as $p \rightarrow \infty$, similarly to $(26)$, we can obtain under $(\mathrm{A}-\mathrm{v})$ and $(\mathrm{A}-\mathrm{vi})$ that

$$
\begin{aligned}
P_{\boldsymbol{\theta}}\left(\boldsymbol{\mu} \in \boldsymbol{R}_{\mathbf{N}}\right) & =P_{\boldsymbol{\theta}}\left(\left\|\boldsymbol{T}_{\mathbf{N}}-\boldsymbol{\mu}\right\|^{2}-\widehat{\Sigma}_{\mathbf{N}} \mid \leq \delta\right) \\
& =P_{\boldsymbol{\theta}}\left(\left\|\boldsymbol{T}_{\mathbf{C}_{L}}-\boldsymbol{\mu}\right\|^{2}-\widehat{\Sigma}_{\mathbf{C}_{L}} \mid \leq \delta\right)+o(1) \geq 1-\alpha+o(1) .
\end{aligned}
$$

It concludes the result.

Acknowledgements Research of the first author was partially supported by Grants-in-Aid for Scientific Research (B) and Challenging Exploratory Research, JSPS, under Contract Numbers 22300094 and 23650142. Research of the second author was partially supported by Grant-in-Aid for Young Scientists (B), JSPS, under Contract Number 23740066.

\section{References}

1. Aoshima M, Yata K (2011a) Two-stage procedures for high-dimensional data. Seq Anal (Editor's special invited paper) 30:356-399

2. Aoshima M, Yata K (2011b) Authors' response. Seq Anal 30:432-440

3. Aoshima M, Yata K (2011c) Effective methodologies for statistical inference on microarray studies. In: Spiess PE (ed) Prostate Cancer - From Bench to Bedside. InTech, pp. $13-32$

4. Aoshima M, Yata K (2013) A distance-based, misclassification rate adjusted classifier for multiclass, high-dimensional data, revised in Ann Inst Statist Math

5. Bai Z, Sarandasa H (1996) Effect of high dimension: by an example of a two sample problem. Stat Sin 6:311-329 
6. Chen SX, Qin YL (2010) A two-sample test for high-dimensional data with applications to gene-set testing. Ann Statist 38:808-835

7. Chiaretti S, Li X, Gentleman R, Vitale A, Vignetti M, Mandelli F, Ritz J, Foa R (2004) Gene expression profile of adult T-cell acute lymphocytic leukemia identifies distinct subsets of patients with different response to therapy and survival. Blood 103:27712778

8. Ghosh M, Mukhopadhyay N, Sen PK (1997) Sequential estimation. Wiley, New York

9. McLeish DL (1974) Dependent central limit theorems and invariance principles. Ann Probab 2:620-628

10. Pollard KS, Dudoit S, van der Laan MJ (2005) Multiple testing procedures: R multitest package and applications to genomics. In: Gentleman R, Carey V, Huber W, Irizarry R, Dudoit $\mathrm{S}$ (ed) Bioinformatics and computational biology solutions using $\mathrm{R}$ and bioconductor. Springer, New York, pp 249-271

11. Srivastava MS (2005) Some tests concerning the covariance matrix in high dimensional data. J Japan Statist Soc 35:251-272

12. Yata K, Aoshima M (2010) Effective PCA for high-dimension, low-sample-size data with singular value decomposition of cross data matrix. J Mult Anal 101:2060-2077

13. Yata K, Aoshima M (2012) Inference on high-dimensional mean vectors with fewer observations than the dimension. Methodol Comput Appl Probab 14:459-476

14. Yata K, Aoshima M (2013). Correlation tests for high-dimensional data using extended cross-data-matrix methodology. J Mult Anal 117:313-331 